\title{
Activation of Hepatic Stellate Cells Requires Dissociation of E-Cadherin-Containing Adherens Junctions with Hepatocytes
}

Hayato Urushima, ${ }^{*}$ Hideto Yuasa, ${ }^{*}$ Tsutomu Matsubara, ${ }^{*}$ Noriyuki Kuroda, ${ }^{\dagger}$ Yaiko Hara, ${ }^{\dagger}$ Kouji Inoue, ${ }^{\ddagger}$ Kenjiro Wake, ${ }^{*} \S$ Tetsuji Sato, ${ }^{\dagger}$ Scott L. Friedman," and Kazuo Ikeda*

From the Department of Anatomy and Regenerative Biology, * Graduate School of Medicine, Osaka City University, Osaka, Japan; the Department of Anatomy, Tissue and Cell Biology, ${ }^{\dagger}$ School of Dental Medicine, Tsurumi University, Yokohama, Japan; the Research Center of Electron Microscopy, ${ }^{\ddagger}$ School of Dental Medicine, Tsurumi University, Yokohama, Japan; the Liver Research Unit, ${ }^{\S}$ Minophagen Pharmaceutical Co., Ltd., Tokyo, Japan; and the Division of Liver Diseases," Icahn School of Medicine at Mount Sinai, New York, New York

Accepted for publication

December 3, 2020.

Address correspondence to Hayato Urushima, Ph.D., or Kazuo Ikeda, M.D., Ph.D., 1-4-3 Asahi-machi, Abenoku, Osaka 545-8585, Japan. E-mail: urushima. hayato@med.osaka-cu.ac.jp orikeda@med.osaka-cu.ac.jp.

\begin{abstract}
Hepatic stellate cells (HSCs) are resident mesenchymal cells in the space of Disse interposed between liver sinusoidal endothelial cells and hepatocytes. Thorn-like microprojections, or spines, project out from the cell surface of HSCs, crossing the space of Disse, to establish adherens junctions with neighboring hepatocytes. Although HSC activation is initiated largely from stimulation by adjacent cells, isolated HSCs also activate spontaneously in primary culture on plastic. Therefore, other unknown HSC-initiating factors apart from paracrine stimuli may promote activation. The dissociation of adherens junctions between HSCs and hepatocytes as an activating signal for HSCs was explored, establishing epithelial cadherin (E-cadherin) as an adhesion molecule linking hepatocytes and HSCs. In vivo, following carbon tetrachloride-induced liver injury, HSCs lost their spines and dissociated from adherens junctions in the early stages of injury, and were subsequently activated along with an increase in YAP/TAZ expression. After abrogation of liver injury, HSCs reconstructed their spines and adherens junctions. In vitro, reconstitution of E-cadherin-containing adherens junctions by forced E-cadherin expression quiesced HSCs and suppressed TAZ expression. Additionally, increase of TAZ expression leading to the activation of HSCs by autocrine stimulation of transforming growth factor- $\beta$, was revealed as a mechanism of spontaneous activation. Thus, we have uncovered a critical event required for HSC activation through enhanced TAZ-mediated mechanotransduction after the loss of adherens junctions between HSCs and hepatocytes. (Am J Pathol 2021, 191: 438-453; https:// doi.org/10.1016/j.ajpath.2020.12.007)
\end{abstract}

Hepatic stellate cells (HSCs) are resident mesenchymal cells localized within the space of Disse, interposed between liver sinusoidal endothelial cells (LSECs) and hepatocytes. Quiescent HSCs store vitamin A lipid droplets in their cytoplasm and encircle the endothelial cells with their long, branching cellular process cells to form hepatic sinusoids. Characteristically, many thorn-like microprojections, or spines, project out from the cell surface of HSCs, crossing the space of Disse, to establish adherens junctions with neighboring hepatocytes. In response to hepatic injury of any etiology, quiescent HSCs transdifferentiate into activated myofibroblasts to produce extracellular matrix (ECM), including collagen and fibronectin, and proliferate, migrate toward regions of injury, and lose vitamin A lipid droplets. After cessation of injury, activated HSCs either undergo apoptosis or revert to an inactivated phenotype, ${ }^{1,2}$ which reduces production of ECM. However, persistent activation of HSCs during chronic liver injury due to viral infection, alcohol, or in nonalcoholic steatohepatitis provokes

\footnotetext{
Supported by JSPS KAKENHI grant JP17K15561, Osaka City University "Think Globally, Act Locally" Research Grant for Young Scientists 2018 (H.U.), NIH grant RO1DK56621 (S.L.F.), and Grant for Research Program on Hepatitis from the Japan Agency for Medical Research and Development (AMED) grant 16fk0210104h0001 (K.I.).

Disclosures: None declared.
} 
accumulation of collagen in the space of Disse, leading to capillarization of sinusoids, followed by panlobular fibrosis. Furthermore, cirrhosis, the most advanced form of fibrosis, is a critical risk factor for hepatic carcinogenesis. ${ }^{3}$ Therefore, clarifying the extracellular signals that suppress activated HSCs is an important step toward identifying novel therapeutic targets for cirrhosis and hepatocellular carcinoma, although none has yet been identified.

Canonically, HSC activation has been conceived as a two-phase event: initiation followed by perpetuation. Initiation refers to early changes in gene expression and phenotype that render cells responsive to stimulants for perpetuation. ${ }^{4}$ The initiation of HSC activation results primarily from paracrine stimulation produced by neighboring cells, including hepatocytes, macrophages, LSECs, inflammatory cells, platelets, and HSCs themselves. ${ }^{5}$ After initiation, several features of the perpetuation phase, including proliferation, ECM production, chemotaxis, and increased contractility may occur, depending on the type of stimulus. For instance, platelet-derived growth factor is the most potent HSC mitogen identified, ${ }^{6,7}$ and transforming growth factor beta (TGF- $\beta$ ) induces the production of collagen and other ECM molecules and down-regulates the degradation of ECM by matrix metalloproteinases in HSCs. ${ }^{8-10}$ However, isolated HSCs activate spontaneously in the absence of these exogenous initiation factors, and culture on uncoated plastic or other stiff matrices. ${ }^{11,12}$ This raises the prospect that initiating factors other than paracrine signals may also contribute to cellular activation.

Although HSCs surround LSECs in normal liver, their contact with LSECs is smooth and does not constitute direct cell adhesion. ${ }^{13}$ Conversely, HSCs extend protrusions called spines, that establish adherens junction with hepatocytes. ${ }^{14}$

The current study focused on the biological significance of contact between HSCs and hepatocytes through these adherens junctions. It was found that early activation of HSCs requires dissociation of these adherens junctions after injury to hepatocytes, which constitutes a critical event required for HSC activation through enhanced mechanotransduction.

\section{Materials and Methods}

\section{Ethics Statement}

All experiments were conducted in compliance with the guidelines for the care and use of laboratory animals and approved by Osaka City University.

\section{Liver Injury Model}

Liver injury was induced by intraperitoneal injections of carbon tetrachloride $\left(\mathrm{CCl}_{4}\right)$ to 6- to 7-week-old Wistar rats or 8- to 10-week-old C57BL/6j mice $(0.5 \mu \mathrm{L} / \mathrm{g}$ body weight, dissolved in corn oil at a ratio of 1:3).
Table 1 Real-Time Quantitative PCR Primer Sequences

\begin{tabular}{|c|c|c|}
\hline Gene name & & Primer sequences \\
\hline \multirow[t]{2}{*}{$\operatorname{Tgfb} 1$} & Forward & 5'-TCGAGGGCGAGAGAAGTTTA-3' \\
\hline & Reverse & 5'-AAAAGAATGTCCCGGCTCTC-3' \\
\hline \multirow[t]{2}{*}{ Pdgfrb } & Forward & 5'-CGCCTGCAAGTGTGAGACAAT-3' \\
\hline & Reverse & 5'-CGAATGGTCACCCGAGCTT-3' \\
\hline \multirow[t]{2}{*}{ Tnfa } & Forward & 5'-TCССAGGTTCTCTTCAAGGGA-3' \\
\hline & Reverse & $5^{\prime}$-GGTGAGGAGCACGTAGTCGG-3' \\
\hline \multirow[t]{2}{*}{ Il1 $b$} & Forward & $5^{\prime}$-TTGACGGACCCCAAAAGATG - $3^{\prime}$ \\
\hline & Reverse & $5^{\prime}-$ TGGACAGCCCAGGTCAAAG- $3^{\prime}$ \\
\hline \multirow[t]{2}{*}{ Il6 } & Forward & $5^{\prime}-$ TGATGCACTTGCAGAAAACA-3' \\
\hline & Reverse & $5^{\prime}$-ACCAGAGGAAATTTTCAATAGGC - ${ }^{\prime}$ \\
\hline \multirow[t]{2}{*}{ Ctgf } & Forward & 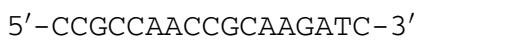 \\
\hline & Reverse & $5^{\prime}-$ ACCGACCCACCGAAGACA- $3^{\prime}$ \\
\hline \multirow[t]{2}{*}{ Acta2 } & Forward & $5^{\prime}$ - CGAAACCACCTATAACAGCATCA-3' \\
\hline & Reverse & $5^{\prime}$-GCGTTCTGGAGGGGCAAT- $3^{\prime}$ \\
\hline \multirow[t]{2}{*}{ Col1a1 } & Forward & $5^{\prime}$-CCAAGGGTAACAGCGGTGAA- $3^{\prime}$ \\
\hline & Reverse & 5'-ССТCGTTTTCСтTCTTCTCCG-3' \\
\hline \multirow[t]{2}{*}{ Col1a2 } & Forward & 5'-TGTTGGCCCATCTGGTAAAGA-3' \\
\hline & Reverse & 5'-CAGGGAATCCGATGTTGCC-3' \\
\hline \multirow[t]{2}{*}{ Cdh1 } & Forward & 5'-GAGGTCTACACCTTCCCGGT-3' \\
\hline & Reverse & 5'-CCACTTTGAATCGGGAGTCT-3' \\
\hline \multirow[t]{2}{*}{ Cdh2 } & Forward & 5'-CAGGGTGGACGTCATTGTAG-3' \\
\hline & Reverse & $5^{\prime}$-AGGGTCTCCACCACTGATTC-3' \\
\hline \multicolumn{3}{|l|}{ Human } \\
\hline \multirow[t]{2}{*}{ TGFB1 } & Forward & $5^{\prime}-\mathrm{CCCTGGACACCAACTATTGC-3^{ \prime }}$ \\
\hline & Reverse & $5^{\prime}$-GCAGAAGTTGGCATGGTAGC-3' \\
\hline \multirow[t]{2}{*}{ PDGFRB } & Forward & 5'-GTGCTCACCATCATCTCCCT-3' \\
\hline & Reverse & 5'-АСТСААТСАССТTССАТCGG-3' \\
\hline \multirow[t]{2}{*}{ CTGF } & Forward & $5^{\prime}$-CAGGCTAGAGAAGCAGAGCC- $3^{\prime}$ \\
\hline & Reverse & 5'-TGGAGATTTTGGGAGTACGG-3' \\
\hline \multirow[t]{2}{*}{ ACTA2 } & Forward & 5'-GACCGAATGCAGAAGGAGAT-3' \\
\hline & Reverse & 5'-CACCGATCCAGACAGAGTATTT-3' \\
\hline \multirow[t]{2}{*}{ COL1A1 } & Forward & $5^{\prime}-$ AAGAGGAAGGCCAAGTCGAG- $3^{\prime}$ \\
\hline & Reverse & 5'-CACACGTCTCGGTCATGGTA-3' \\
\hline \multirow[t]{2}{*}{ COL1A2 } & Forward & $5^{\prime}$-GAAAAGGAGTTGGACTTGGC-3' \\
\hline & Reverse & 5'-AGCAGGTCCTTGGAAACCTT-3' \\
\hline \multirow[t]{2}{*}{$\mathrm{CDH} 1$} & Forward & 5'-GGGTGACTACAAAATCAATC-3' \\
\hline & Reverse & 5'-GGGGGCAGTAAGGGCTCTTT-3' \\
\hline \multirow[t]{2}{*}{$\mathrm{CDH} 2$} & Forward & $5^{\prime}-$ CACTGCTCAGGACCCAGAT-3' \\
\hline & Reverse & $5^{\prime}-$ TAAGCCGAGTGATGGTCC-3' \\
\hline \multirow[t]{2}{*}{ SERPINH1 } & Forward & $5^{\prime}$-AGAGTAGAATCGTGTCGCGG-3' \\
\hline & Reverse & $5^{\prime}$-CTGAGAAGCAGGAGGGAGC- $3^{\prime}$ \\
\hline \multirow[t]{2}{*}{ SERPINE1 } & Forward & 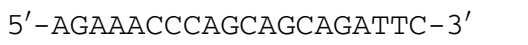 \\
\hline & Reverse & 5'-TGGTGCTGATCTCATCCTTG-3' \\
\hline \multirow[t]{2}{*}{ SNAI1 } & Forward & $5^{\prime}-$ ACCCCAATCGGAAGCCTAACT-3' \\
\hline & Reverse & 5'-GGTCGTAGGGCTGCTGGAA-3' \\
\hline MMP2 & Forward & 5'-TGATGTCCAGCGAGTGGAT-3' \\
\hline & Reverse & 5'-GGAAAGCCAGGATCCATTTT-3' \\
\hline TGFBR2 & Forward & $5^{\prime}$-AGCATCACGGCCATCTGTG-3' \\
\hline & Reverse & $5^{\prime}-$ TGGCAAACCGTCTCCAGAGT-3' \\
\hline
\end{tabular}

\section{Transmission Electron Microscopy}

The tissue blocks prepared with perfusion fixation method were postfixed with phosphate-buffered $2 \%$ osmium tetroxide, dehydrated, and embedded in Poly/Bed 812. Thin sections were examined in a transmission electron 


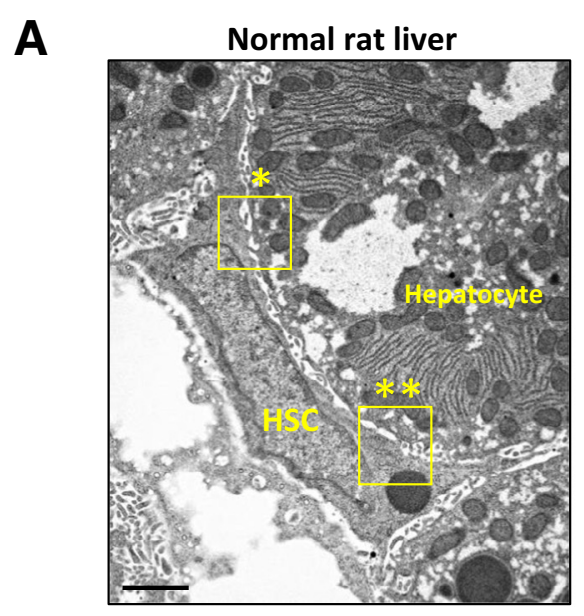

B

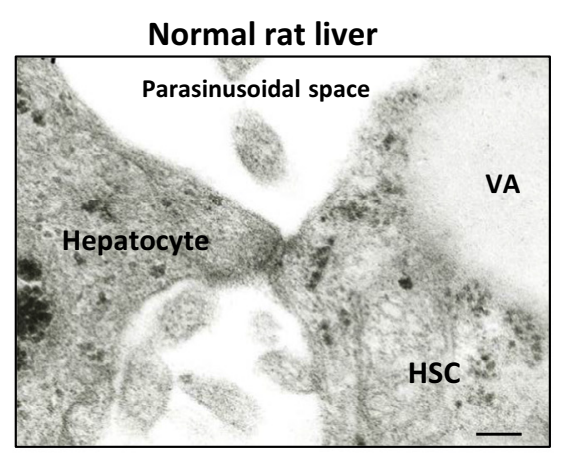

D

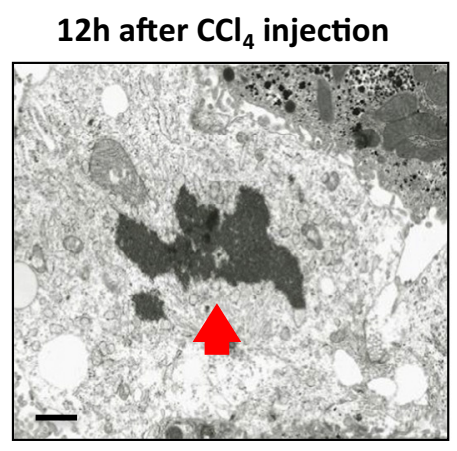

$F$
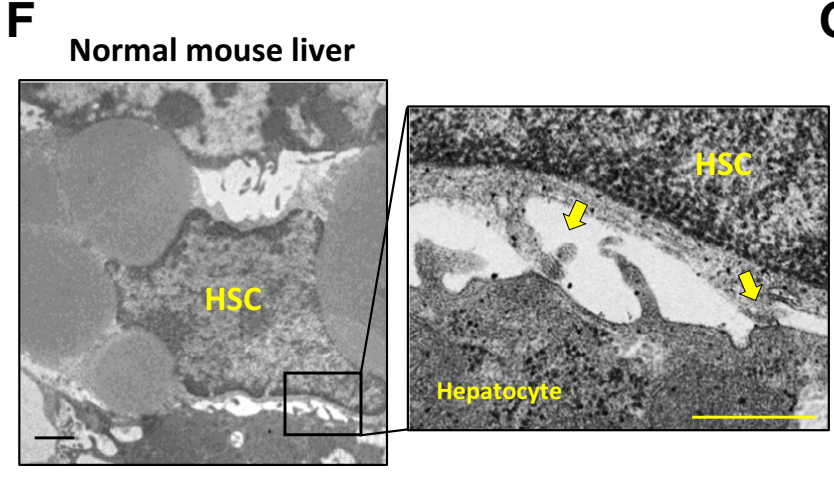

$*$

C

E
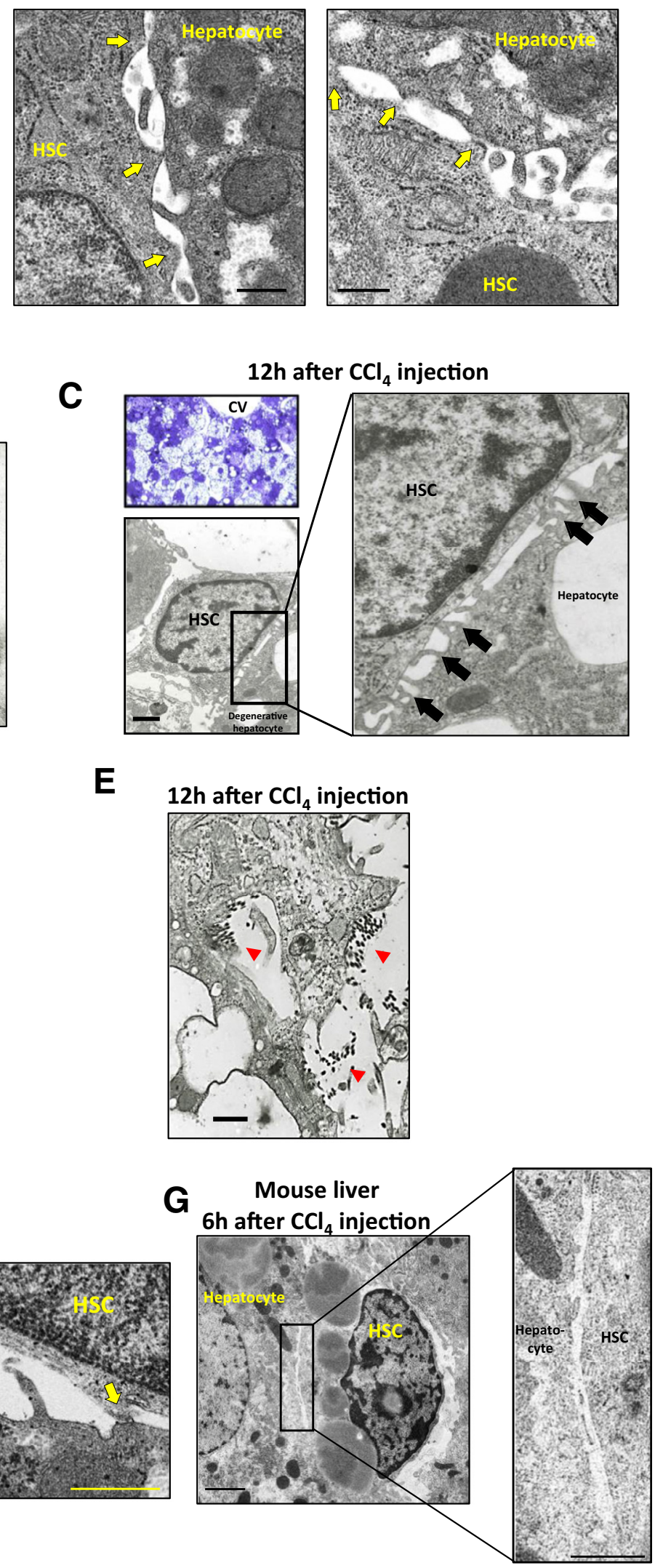
microscope (model 100CX, JEOL Ltd., Tokyo, Japan) with an accelerating voltage of $80 \mathrm{kV}$.

\section{HSC Isolation and Culture}

HSCs were isolated as previously described ${ }^{15}$ from C57BL/ $6 \mathrm{j}$ mice by using pronase/collagenase perfusion followed by Nycodenz gradient centrifugation. Isolated primary HSCs were cultured in Dulbecco's modified Eagle medium (Wako, Osaka, Japan) containing 10\% fetal bovine serum, $100 \mathrm{mg} / \mathrm{mL}$ penicillin, and $100 \mathrm{U} / \mathrm{mL}$ streptomycin.

The human HSC line HHSteCs (lot number 10326) was purchased from ScienCell Research Laboratories (Carlsbad, CA). These cells were maintained by using the Stellate Cell Medium set (catalog number 5301) at $37^{\circ} \mathrm{C}$ in a humidified $5 \%$ carbon dioxide atmosphere.

To generate an epithelial cadherin (E-cadherin)-coated dish, recombinant E-cadherins $(1.5 \mu \mathrm{g} / \mathrm{mL}$; R\&D Systems, Minneapolis, MN) were coated in a 24-well plate with phosphate-buffered saline for 2 hours followed by blocking with $1 \%$ bovine serum albumin containing phosphatebuffered saline for 3 hours. Mouse primary HSCs or HHSteCs were then cultured in the plate.

\section{Three-Dimensional Spheroid Culture}

HHSteCs or mouse primary HSCs were seeded $5.0 \times 10^{3}$ per well in a 96-well, low attachment spheroid culture plate (Sumitomo Bakelite, Tokyo, Japan). For analysis, at least six spheroids were pooled. For neutralization of E-cadherin, Ecadherin monoclonal antibody (SHE78-7, Thermo Fisher Scientific, Waltham, MA) was added into the culture medium at the concentration of $10 \mu \mathrm{g} / \mathrm{mL}$. For the analysis of TGF- $\beta$ signaling in the presence or absence of E-cadherin-mediated adherens junctions, empty vector-transfected (control), or E-cadherin overexpressed HHSteC cells were cultured as spheroid and stimulated by $10 \mathrm{ng} / \mathrm{mL}$ of TGF- $\beta$. Proteins were then isolated at $0,5,10,30$, and 60 minutes after TGF- $\beta$ stimulation.

\section{Plasmid Transfection}

To generate transient overexpression of E-cadherin or TAZ, HHSteCs were transfected with an empty plasmid, hE-
cadherin-pcDNA3 (gift from Barry Gumbiner; plasmid number 45769, Addgene, Cambridge, MA) or HA-TAZ (gift from Kunliang Guan; plasmid number 32839, Addgene) using lipofectamine 3000 (Thermo Fisher Scientific) following the manufacturer's protocol.

\section{Quantitative Real-Time and Direct Reverse Transcription PCR of mRNA}

RNA was extracted from cells by using TRIzol reagent (Thermo Fisher Scientific) and Direct-zol RNA MiniPrep (Zymo Research, Irvine, CA). Real-time quantitative PCR (qPCR) was performed by using cDNA generated from RNA and the SuperScript III Reverse Transcriptase kit (Thermo Fisher Scientific). The qPCR reaction was conducted by using the SYBR green PCR master mix (Thermo Fisher Scientific) in the Thermal Cycler Dice Real Time System 2 (Takara Bio, Shiga, Japan). The values were quantified by using the comparative CT method and were normalized to $18 \mathrm{~S}$ ribosomal RNA. Data are expressed as the ratio to the average of the control group. The primers used in this study are listed in Table 1.

\section{Western Blot}

Cells were homogenized with radioimmunoprecipitation assay buffer $(50 \mathrm{mmol} / \mathrm{L}$ Tris- $\mathrm{HCl}$ at $\mathrm{pH} 7.5,150 \mathrm{mmol} / \mathrm{L}$ $\mathrm{NaCl}, 1 \%$ Triton $\mathrm{X}-100,1 \%$ SDS) containing the protease inhibitor cocktail cOmplete Mini (Roche, Basel, Switzerland) and phosphatase inhibitors $(1 \mathrm{mmol} / \mathrm{L}$ sodium fluoride, $1 \mathrm{mmol} / \mathrm{L} \beta$-glycerol phosphate, and 1 $\mathrm{mmol} / \mathrm{L}$ sodium vanadate). Protein samples were subjected to $8 \%$ to $15 \%$ SDS-PAGE and were transferred to polyvinylidene difluoride membranes using standard Western blot techniques. After blocking with 5\% skim milk, the membranes were probed with primary antibodies diluted at 1:1000 and horseradish peroxidase-conjugated secondary antibodies diluted at 1:5000. Immunoreactive bands were visualized by using the ImmunoStar Zeta or ImmunoStar LD system and were detected with an LAS3000 or LAS4000 device (GE Healthcare, Milwaukee, WI). WB Stripping Solution (Nacalai Tesque, Kyoto, Japan) was used to remove the antibodies from the Western blot

\footnotetext{
Figure 1 Morphologic changes of spine-mediated adherens junctions in hepatic stellate cells (HSCS) with hepatocytes during liver inflammation. A-E: Transmission electron microscopy of liver in 6- to 7-week-old Wistar rats. A: A representative image of the relationship between hepatocytes and HSCs in a normal liver is shown in the left panel. The middle and right panels show the enlarged images of the asterisk-marked region of the left panel. HSCs extend spines toward hepatocytes (yellow arrows). Some tips of spines have high electron density, suggesting that the spine constitutes an adherens junction with hepatocytes. B: Shown is the enlarged image of the region where a hepatocyte and an HSC are in contact. C: The top left panel shows the toluidine blue staining image around a central vein (CV) under a light microscope. The representative image around a $\mathrm{CV}$ at 12 hours after carbon tetrachloride (CCl $)$ injection is shown in the bottom left panel. The right panel shows the enlarged figure of the bottom left panel. The spines in HSCs have disappeared while the microvilli extending from the hepatocytes remain (black arrows). D and E: Features of the perpetuation phase of $\mathrm{HSCs}_{\text {at }} 12$ hours after $\mathrm{CCl}_{4}$ injection. D: A mitotic figure of the HSC is shown by a red arrow. E: De novo synthesis of collagen fibrils by the HSC are shown by red arrowheads. F-G: Transmission electron microscopy of normal mouse liver. F: A representative image of the relationship between hepatocytes and HSCs in a normal liver of 8- to 10-week-old C57BL/ $6 \mathrm{j}$ mice is shown in the left panel. The enlarged image focusing on the site of adherens junctions between hepatocytes and HSCs (yellow arrows) is shown in the right panel. G: The representative image of the mouse liver at 6 hours after $\mathrm{CCl}_{4}$ injection is shown in the left panel. The right panel shows the enlarged image of the loss of adherens junctions in HSCs with hepatocytes. Scale bars: $2 \mu \mathrm{m}$ (A and $\mathbf{G}$, left panels) and $500 \mathrm{~nm}$ (A, middle and right panels; E; and $\mathbf{F}$, right panel); $100 \mathrm{~nm}(\mathbf{B}) ; 1 \mu \mathrm{m}$ (C and D; F, left panel; and G, right panel). VA, vitamin A droplet.
} 
A

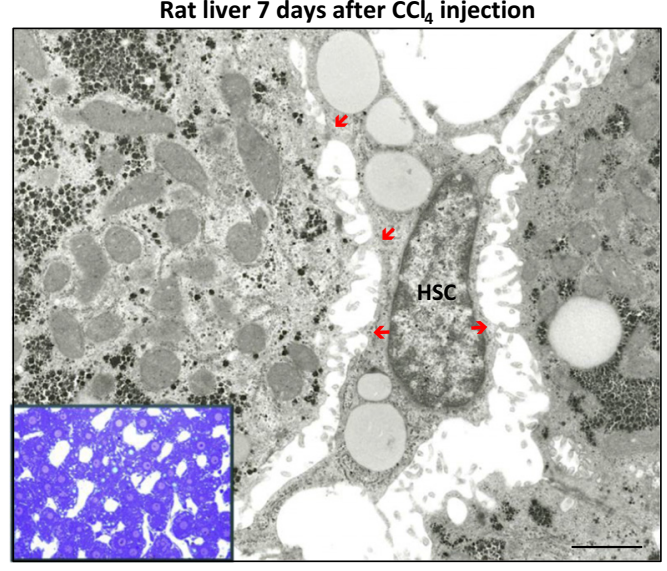

C

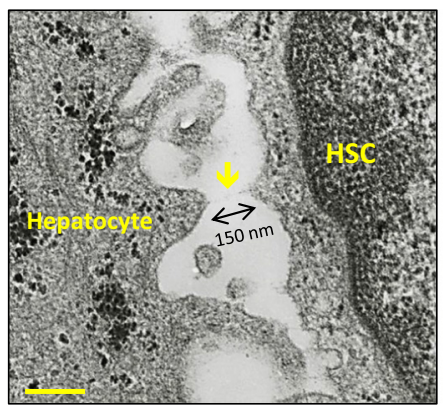

B

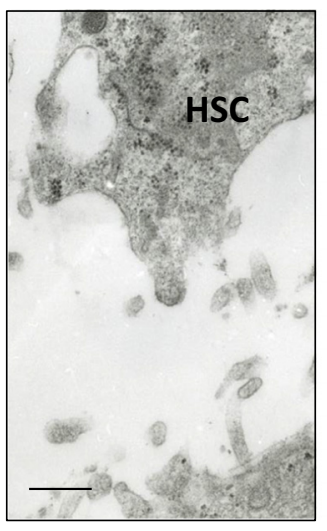

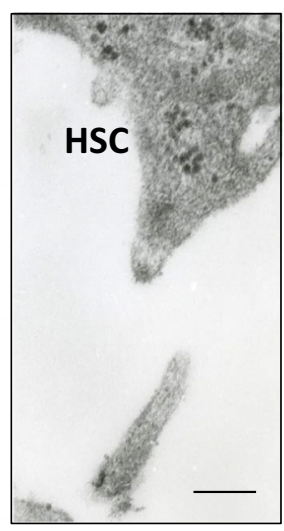

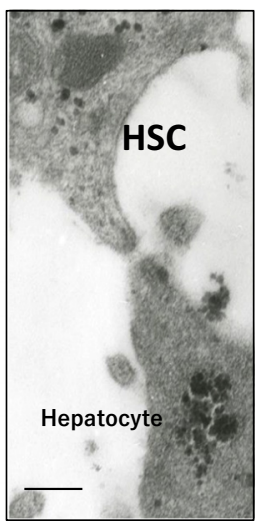

Figure 2 Hepatic stellate cells (HSCs) reconstruct adherens junctions with regenerated hepatocytes in the recovery stage. Transmission electron microscopy of the liver of 6- to 7-week-old Wistar rats 7 days after carbon tetrachloride $\left(\mathrm{CCl}_{4}\right)$ injection. A: HSCs store lipid droplets as a feature of quiescent and reconstructed adherens junctions with hepatocytes (red arrows). The inset figure shows the toluidine blue staining image under a light microscope. B: An HSC, which has not yet recovered the adhesion with hepatocytes, extends regenerating spines and establishes an adherens junction de novo with a regenerating, mitotic hepatocyte. C: Fibrous structure linking a hepatocyte and an HSC is shown as a yellow arrow. An enlarged image is shown in the right panel. Scale bars: $1 \mu \mathrm{m}$ (A); $100 \mathrm{~nm}$ (B); $200 \mathrm{~nm}$ (C).

membrane. The quantification of Western blot bands was performed by using ImageJ software version $1.52 \mathrm{n}(\mathrm{NIH}$, Bethesda, MD; http://imagej.nih.gov/ij). Data are expressed as means \pm SEM of at least three different experiments.

\section{Flow Cytometry}

Isolated HSCs were stained with Alexa Fluor 647 antihuman/mouse CD324 (DECMA-1; BioLegend, San Diego, CA) or isotype control (RTK2071; BioLegend). HSCs emit autofluorescence under UV excitation due to their vitamin A content. ${ }^{16}$ Therefore, the expression of E-cadherin in UV autofluorescence-positive cells was characterized by using FACS LSR II (BD Biosciences, San Diego, CA).

\section{Histological, Immunohistochemical, and Immunofluorescence Analysis}

For histological evaluation, mouse liver were fixed with $4 \%$ paraformaldehyde and embedded into paraffin. Then, mouse liver section were sliced $(4 \mu \mathrm{m})$ and stained with hematoxylin and eosin. For immunohistochemical analysis, they were deparaffinized, hydrated, heated to $110^{\circ} \mathrm{C}$ in citrate buffer for 20 minutes, depleted of endogenous peroxidase activity, and then blocked with Blocking One (Nacalai
Tesque) for 1 hour. Next, the slides were treated with primary antibodies overnight at $4^{\circ} \mathrm{C}$. The slides were incubated with secondary antibody (Bioss, Inc., Woburn, MA) for 1 hour at room temperature, and the reaction was visualized by diaminobenzene substrate (Vector Laboratories, Burlingame, CA). All specimens were counterstained with hematoxylin. For immunofluorescence analysis, after hybridization with primary antibodies, the slides were incubated with Alexa Fluor 488 or 594 conjugated donkey anti-mouse or rabbit IgG (Invitrogen, Carlsbad, CA). DAPI (Dojindo, Kumamoto, Japan) was used as a nuclear marker. For quantitative analysis, three images were taken at every indicated time point, and cells positive for Ly6G per field of each view were counted.

For immunoelectron microscopy, $4 \%$ paraformaldehyde fixative-fixed, frozen mouse liver slices were cut into $4 \mu \mathrm{m}$ with a cryostat (HM 560; Microm, Walldorf, Germany). The immunostaining procedure was similar to that described in the previous paragraph. After visualizing by diaminobenzene, they were postfixed with $2 \%$ osmium tetroxide (Wako) for 1 hour on ice and dehydrated in an ethanol series before embedding in a Quetol 812 mixture. Ultrathin sections were stained with lead citrate solution and observed in Talos F200C G2 (Thermo Fisher Scientific) with an accelerating voltage of $200 \mathrm{kV}$. 


\section{Primary Antibodies}

The following antibodies were used for Western blot analysis and immunofluorescence: anti-E-cadherin (24E10), anti-phospho-Smad2 (Ser465/467), anti-Smad2 (D43B4), anti-YAP (D8H1X), anti-TAZ (V386), and anti-platelet-derived growth factor-receptor $\beta$ (28E1), purchased from Cell Signaling Technology (Danvers, MA). Anti-neural cadherin (H-2), anti-placental cadherin (D-6), anti-vascular endothelial cadherin (F-8), and anti-heat shock protein 47 (G-12) were purchased from Santa Cruz Biotechnology (Santa Cruz, CA). Anti- $\alpha-$ smooth muscle actin (1A4; DAKO, Glostrup, Denmark), anti-Ly6G (1A8; R\&D systems), anti-glyceraldehyde-3phosphate dehydrogenase (Millipore, Billerica, MA), and anti-cytoglobin (kindly provided by Prof. Norifumi Kawada, Osaka City University) were also used for Western blot and immunohistochemical analyses.

\section{ELISA of Cellular Supernatants}

E-cadherin or TAZ was overexpressed in HHSteC cells and cultured as spheroids. Forty-eight hours later, the levels of TGF- $\beta$ in the supernatant of spheroid culture were determined by using a human TGF- $\beta$ ELISA kit (BioLegend).

\section{Statistical Analysis}

All data are expressed as means $\pm \mathrm{SD}$. All data were analyzed by using analysis of variance with a post hoc Dunnett's test or unpaired $t$-test.

\section{Results}

\section{Activated HSCs Lose Adherens Junctions with Hepatocytes}

The morphologic changes of adherens junctions between hepatocytes and $\mathrm{HSCs}$ in rat liver during $\mathrm{CCl}_{4}$-induced acute liver injury were investigated first. In normal liver, several spine-mediated adherens junctions with hepatocytes projected out both from the surface of the dendritic processes as well as from the cell bodies (Figure 1A). A high magnification of an adherens junction between the spine of an HSC and hepatocyte is shown in Figure 1B. The adhesive power of the junction is illustrated by the subjunctional cytoplasm of the hepatocyte being pulled up to form a spine. Actin-like microfibrils in both cells aligned together within each subjunctional region.

At 12 hours, individual HSCs in the injured areas were surrounded by hepatocytes with ballooning degeneration (upper left image of Figure 1C). Some microvilli of the degenerating hepatocytes remained in close contact with HSCs. Importantly, however, neither spines nor adherens junctions were observed (Figure 1C), whereas mitotic figures (Figure 1D) were observed in HSCs.
Characteristically, Golgi vacuoles located close to the cell membrane of HSCs were filled with collagen fibrils, some of which appeared to be secreted into the extracellular space (Figure 1E).

Based on the findings from the rat experiments, morphologic studies were validated in a mouse $\mathrm{CCl}_{4}$ model. Similar to findings in rat liver, spine-mediated adherens junctions with hepatocytes were also identified between hepatocytes and HSCs in normal mouse liver (Figure 1F), which disappeared within 6 hours after $\mathrm{CCl}_{4}$ injection (Figure 1G).

\section{HSCs Reconstitute Adherens Junctions with Regenerated Hepatocytes during Recovery}

Next examined was the relationship between HSCs and hepatocytes in rat liver 7 days after a single $\mathrm{CCl}_{4}$ injection, at the stage of subsiding injury. Damaged hepatocytes had recovered, and inflammatory cell infiltration had subsided. HSCs reconstituted their adherens junctions with hepatocytes with increased lipid droplets, a feature of HSC quiescence $^{4}$ (Figure 2A). Interestingly, the HSCs which had not yet restored their adhesion to hepatocytes, extended regenerating spines and established an adherens junction de novo with a regenerating, mitotic hepatocyte (Figure 2B). In the same region, fibrous structures linking hepatocytes and HSCs were observed at the tip of the spine (Figure 2C).

\section{E-Cadherin Mediates Adhesion between Hepatocytes and HSCS}

Cadherins constitute a large superfamily of cell-cell adhesion molecules. ${ }^{17,18}$ The classic cadherins consist of Ecadherin, neural cadherin, placental cadherin, and vascular endothelial cadherin. ${ }^{19}$ Thus, we explored the cadherin(s) responsible for hepatocyte-HSC adherens junctions. Almost all of the isolated mouse HSCs contained lipid droplets (left upper panel of Figure 3A) and expressed cellspecific markers such as cytoglobin ${ }^{20}$ and heat shock protein $47,{ }^{21}$ whereas none of the HSCs expressed of albumin and CK18, the specific markers of hepatocytes (right upper panel of Figure 3A). These data confirmed the high purity of isolated mouse HSCs. In addition, murine HSCs expressed E-cadherin but not other cadherins (bottom panel, Figure 3A). The expression of E-cadherin in HSCs was examined by flow cytometry. Because they contain vitamin A, quiescent HSCs have autofluorescence when excited by UV. ${ }^{22}$ As shown in the upper panel of Figure 3B, $99.1 \%$ of isolated cells were UV-positive, which expressed E-cadherin (middle and bottom panel, Figure 3B). Similar to a previous report of rat HSCs, ${ }^{12}$ this study also confirmed that the expression of cadherin in mouse HSCs switched from Ecadherin to neural cadherin along with morphologic changes and spontaneous activation (Figure 3C). Moreover, 
A
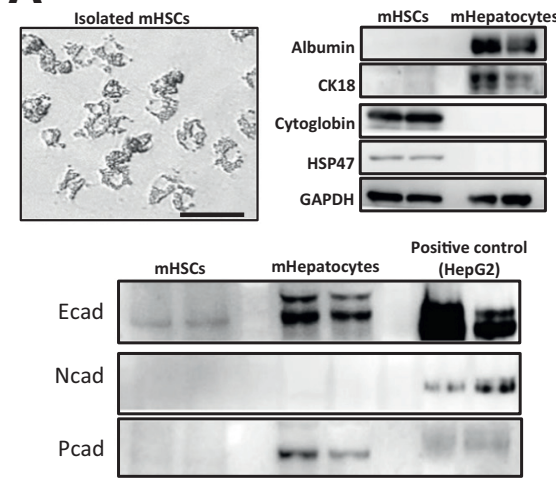

VEcad

GAPDH

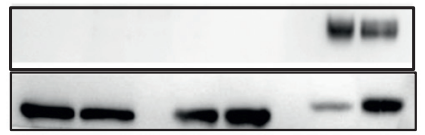

B
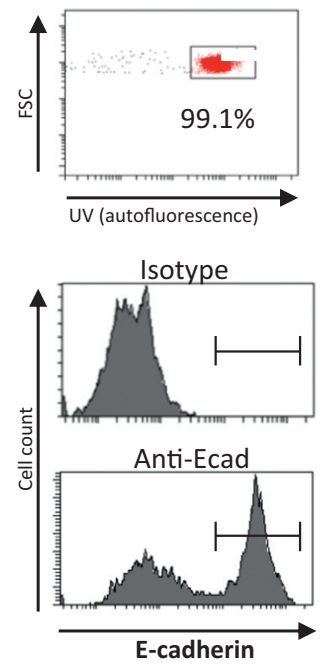

C
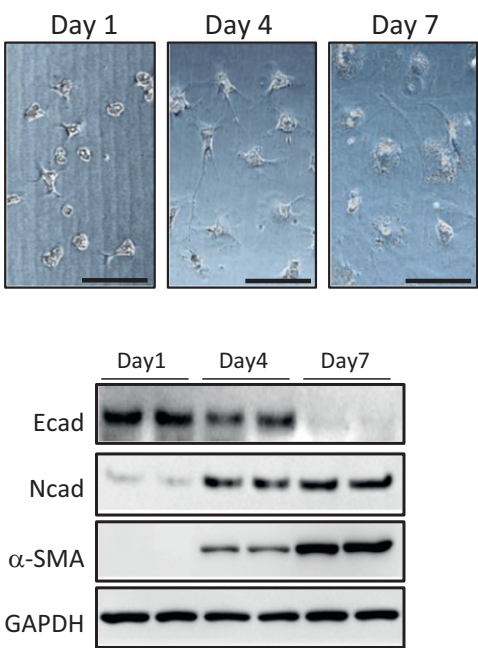

D

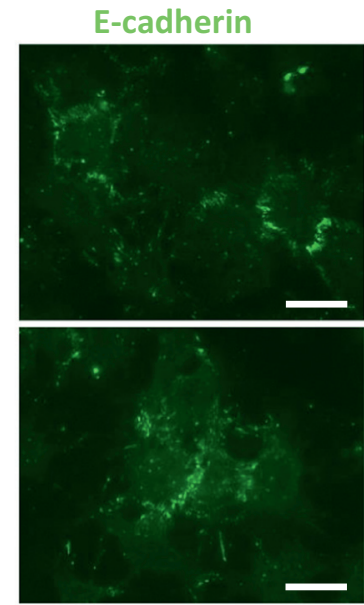

E-cadherin

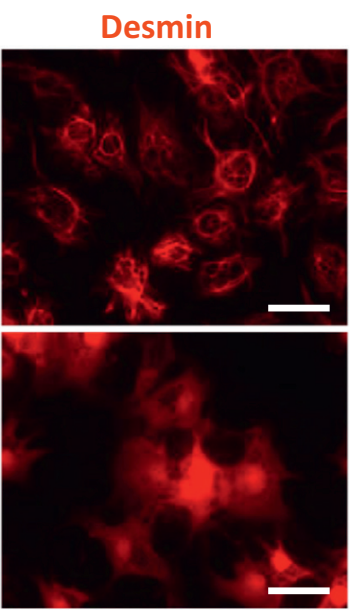

Cytoglobin
DAPI

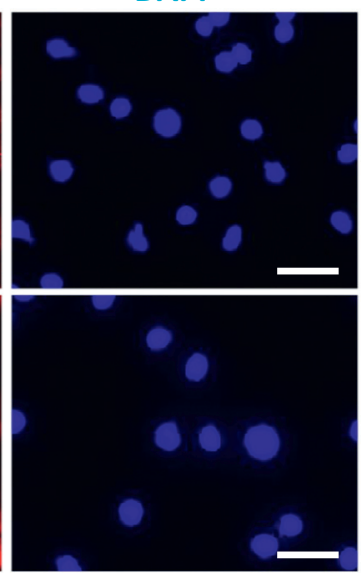

DAPI
Merge

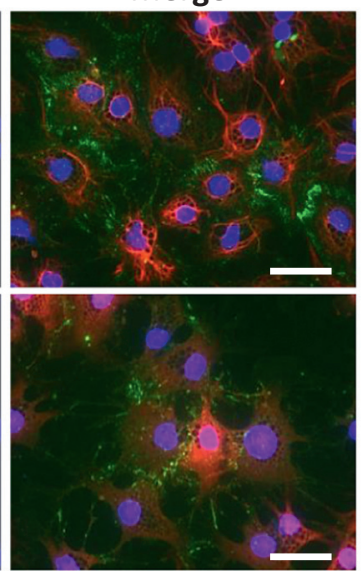

Merge

E

E-cadherin/DAPI

Cytoglobin/DAPI

Merge
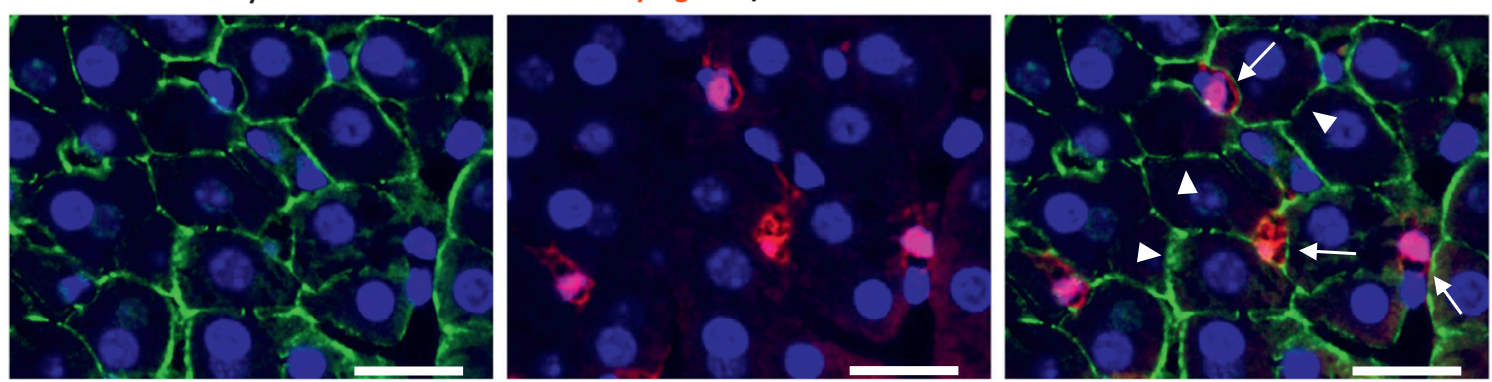

$\mathbf{F}$

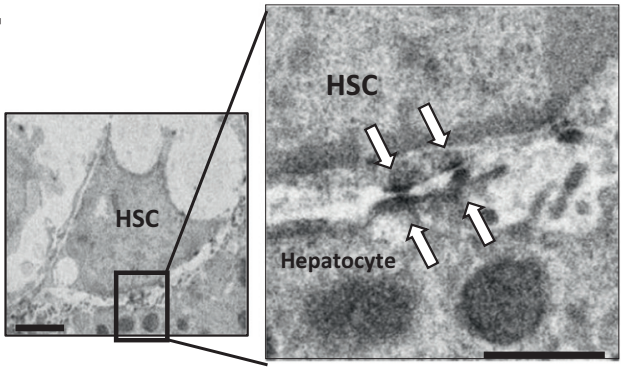

G

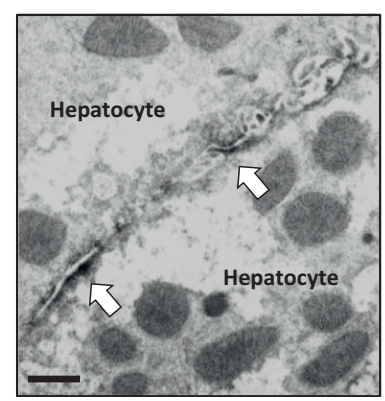


desmin $^{23}$ or cytoglobin-positive HSCs expressed E-cadherin by immunofluorescence (Figure 3D).

Generally, E-cadherin is expressed primarily in epithelial cells, including hepatocytes. Although HSCs are a type of mesenchymal cells, previous studies have shown that Ecadherin co-localizes with glial fibrillary acidic protein, a classic marker of quiescent HSCs in rat normal liver. ${ }^{12,24}$ Therefore, we assessed immunohistochemical staining for E-cadherin in normal mouse liver, and detected it at the surrounding cytoglobin-positive cells as well as between hepatocytes (Figure 3E), indicating that hepatocytes express E-cadherin even on the sinusoidal surface. Considering that cadherins are homophilic cell-adhesion molecules, these findings indicate that E-cadherin might contribute to the adhesion between hepatocytes and HSCs.

For a more detailed analysis, the localization of E-cadherin was visualized by using immunoelectron microscopy. As expected, the immunostain for E-cadherin was confirmed at the adherens junctions between hepatocytes and HSCs (Figure 3F) as well as hepatocyte-hepatocyte adherens junctions (Figure 3G). Taken together, our findings support the conclusion that E-cadherin is expressed in HSCs and mediates adhesion with hepatocytes.

\section{Initiation of HSC Activation Is Independent of Stimulation by Exogenous Initiation Factors}

We next analyzed the temporal relationship between the disappearance of the adherens junctions and the enhancement of exogenous factors for HSC initiation using a mouse $\mathrm{CCl}_{4}$ model. Hematoxylin and eosin staining and immunohistochemical staining for a neutrophil marker, Ly6G, revealed that the infiltration of neutrophils, which secrete exogenous activation factors of HSCs such as reactive oxygen species, ${ }^{25}$ was present at 12 and 24 hours but not at 6 hours after $\mathrm{CCl}_{4}$ when E-cadherin-mediated adherens junctions disappeared (Figure 4, A and B). As indicated by qPCR, mRNA expression levels in the liver for exogenous stimuli that encode important drivers of HSC activation, including Tgfbl, Pdgf, Tnfa, ${ }^{26} I l l b,{ }^{27,28}$ and $I l 6,{ }^{29}$ were still not changed within 6 hours after $\mathrm{CCl}_{4}$ injection (Figure $4 \mathrm{C}$ ). In addition, the expression of Acta2, which encodes $\alpha$ smooth muscle actin, a specific marker of HSC activation, tended to increase 3 hours after $\mathrm{CCl}_{4}$ injection.

The rodent experiments revealed that the HSCs had already lost adherens junctions with hepatocytes in the early stage of injury, when few, if any, exogenous initiating factors are produced. However, TGF- $\beta$ signaling, which is a typical driver of perpetuation of the HSC-activated phenotype, is not always regulated by its level of expression but rather through its activation from its latent form. We therefore analyzed the effect of E-cadherin-mediated adherens junctions on the response to TGF- $\beta$ signaling. Ecadherin-overexpressed HHSteCs were cultured as spheroids that reconstruct E-cadherin-mediated adherens junctions and were stimulated by TGF- $\beta$. Phase-contrast microscopy showed a clear outline of each cell in the control. In contrast, a fused image of the cell contour was observed after overexpression of E-cadherin. In addition, whereas the expression of TGF- $\beta$ receptor type II was not altered (Figure 4D), phosphorylation of Smad2 and Smad3 was suppressed in HHSteCs compared with control cells after reconstitution of E-cadherin-mediated adherens junctions (Figure 4E). Additionally, the expression of $S N A I 1$ and $C D H 2$ genes and $\alpha$-smooth muscle actin protein, which are downstream of TGF- $\beta$ signaling, was significantly decreased in the presence of homophilic E-cadherin binding (Figure 4, F and G). These findings support our hypothesis that the initiation of HSCs by the disappearance of E-cadherin-mediated adherens junctions occurred independently from the stimulation by exogenous initiation factors.

\section{Mechanism of HSC Initiation following the Loss of Adherens Junctions}

We next investigated the pathway initiating activation of HSCs during the loss of adherens junctions with hepatocytes. In

\footnotetext{
Figure 3 Epithelial cadherin (E-cadherin) mediates adhesion between hepatocytes and hepatic stellate cells (HSCs). A: The image of the phase-contrast microscope of isolated mouse HSCs (mHSCs) is shown in the top left panel. The high purity of isolated mHSCs was confirmed by Western blot (top right panel). Western blot analysis of cadherins in $\mathrm{mHSC}$ just after isolation is shown in the bottom panel. Two representatives are shown here. HepG2 is positive control for the cadherins. Albumin and cytokeratin 18 (CK18) are specific markers of hepatocytes. Cytoglobin and heat shock protein 47 (HSP47) are specific markers of HSCs. B: Flow cytometry analysis of primary mouse HSCs. HSCs are gated by UV-positive cells (top panel). Histogram data show the expression of Ecadherin in primary mouse HSCs. A representative image of at least three independent experiments is shown. HSCs were stained with isotype antibody (middle panel) or anti-E-cadherin antibody (bottom panel). C: Isolated mHSCs were cultured and observed by phase-contrast microscopy at the indicated time points. HSCs on day 1 contain abundant lipid droplets. However, the amount of lipid droplets was decreased, and the cell bodies began spreading with time. The bottom panel shows Western blot analysis of cultured mHSCs at the indicated time points. Representative images of three independent experiments are shown. D: Isolated mHSCs were cultured on glass chamber slides. After 24 hours' culture, HSCs were immunostained for E-cadherin and quiescent HSC markers desmin and cytoglobin for 24 hours followed by DAPI staining for 15 minutes. E: Normal mouse liver sections were immunostained for E-cadherin and cytoglobin, a specific marker of HSCs, for 24 hours followed by DAPI staining for 15 minutes. E-cadherin was detected by immunostaining at the surrounding cytoglobin-positive cells (arrows) as well as between hepatocytes (arrowheads). F-G: Immunoelectron microscopic images of E-cadherin in normal mouse liver. The expression of E-cadherin was confirmed at the adherens junctions between hepatocytes and HSCs (F, arrows) as well as hepatocyte-hepatocyte adherens junctions (G, arrows). $n=4$ (A and $\mathbf{B})$. Scale bars: $50 \mu \mathrm{m}(\mathbf{A} ; \mathbf{C} ; \mathbf{D}$, top panels; and $\mathbf{E}) ; 2 \mu \mathrm{m}$ (F, left panel), $1 \mu \mathrm{m}(\mathbf{F}$, right panel; and $\mathbf{G})$. Ecad, epithelial cadherin; FSC, forward scatter; GAPDH, glyceraldehyde-3-phosphate dehydrogenase; Ncad, neural cadherin; Pcad, placental cadherin; VEcad, vascular endothelial cadherin.
} 
A

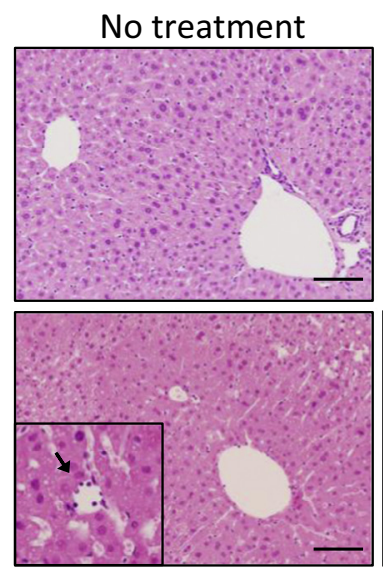

$12 \mathrm{~h}$
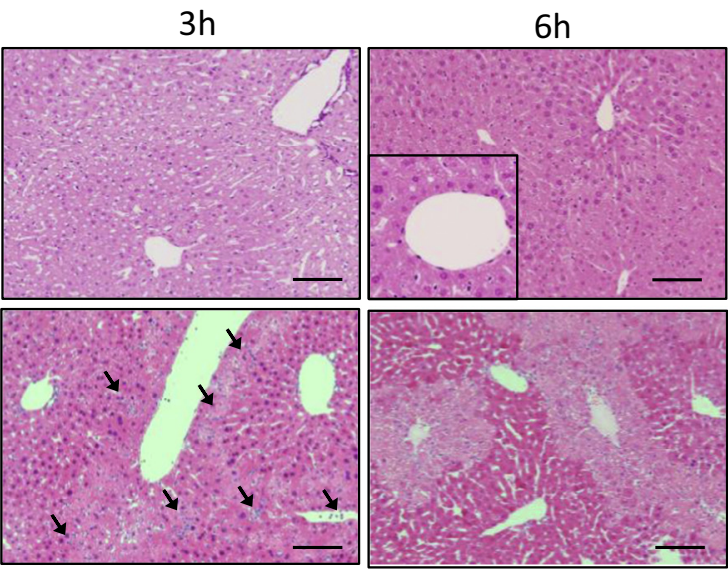

$24 h$

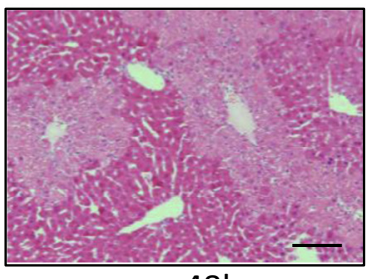

$48 \mathrm{~h}$

C

B

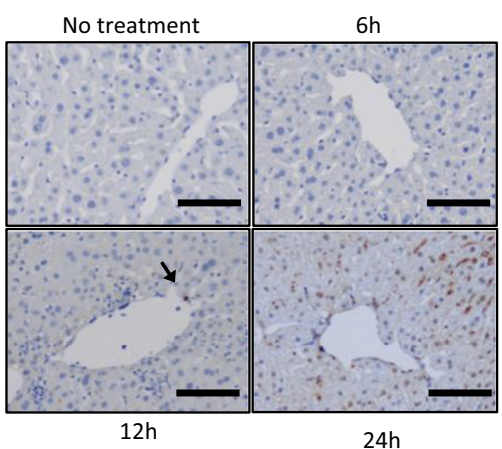

D
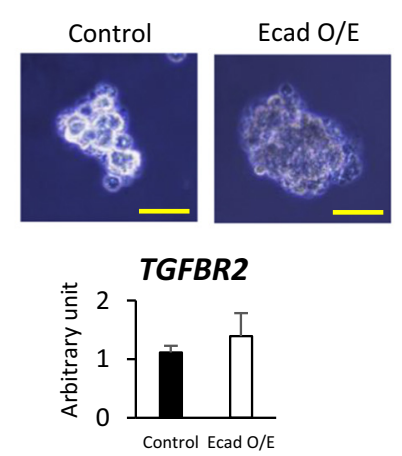

$\mathbf{F}$
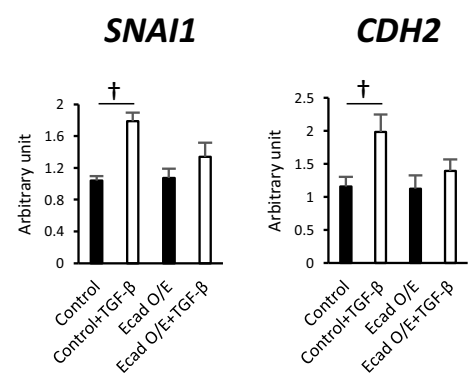

Ly6G positive cells

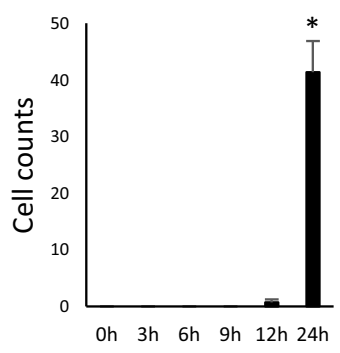

E

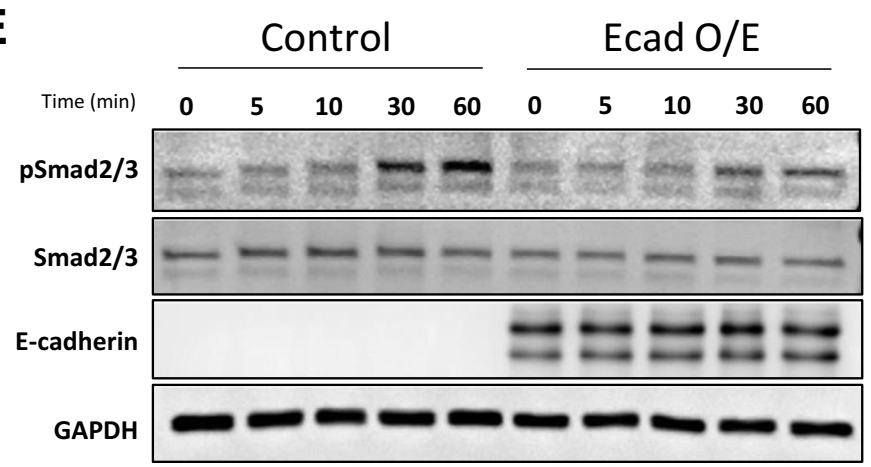

G

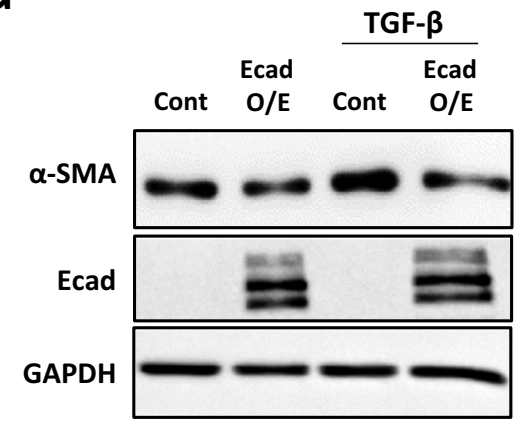

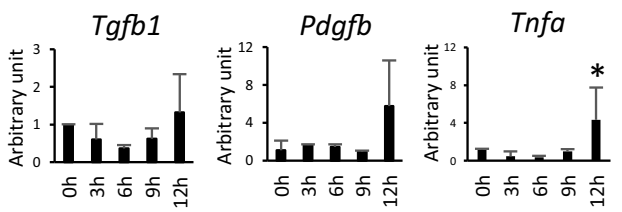

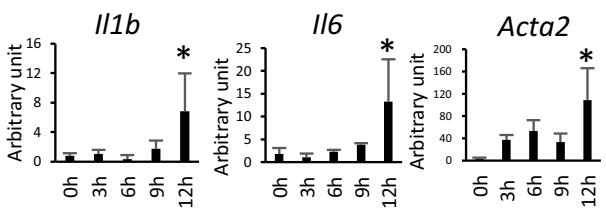


normal differentiated epithelial tissues, contact inhibition suppresses cell activity through homophilic E-cadherin binding to tightly regulate tissue morphogenesis, ${ }^{30,31}$ which involves YAP/TAZ signaling. ${ }^{32}$ When epithelial cells proliferate, YAP/ TAZ localizes to the nucleus where it is transcriptionally active. In contrast, when the epithelial cells contact other cells through E-cadherin, YAP/TAZ is phosphorylated, underlying the effect of contact inhibition. ${ }^{32}$ Immunohistochemical staining for YAP and TAZ revealed that TAZ expression around central veins was increased in mouse liver 12 hours after a $\mathrm{CCl}_{4}$ injection compared with that in normal mouse liver. However, most of the staining was cytoplasmic, not nuclear, and not transcriptionally active (Figure 5A). Moreover, although the expression of TAZ was not observed in desmin-positive cells in untreated mouse liver, TAZ expression increased and localized in the nuclei of desmin-positive cells 12 hours after $\mathrm{CCl}_{4}$ injection (Figure 5B).

We next examined the YAP/TAZ pathway in isolated HSCs from mouse liver 12 hours after $\mathrm{CCl}_{4}$ injection. First, we verified the purity of isolated HSCs from damaged liver after $\mathrm{CCl}_{4}$ injection. As shown in the upper panel of Figure $5 \mathrm{C}$, the expression of cytoglobin, but not albumin, was confirmed in $\mathrm{HSCs}$ from both untreated and $\mathrm{CCl}_{4^{-}}$ injected mice. We then investigated YAP and TAZ expression in HSCs by Western blot analysis. YAP and especially TAZ expression was dramatically increased, along with $\alpha$-smooth muscle actin, which is an activation marker of HSCs, compared with those of untreated mice (bottom panel of Figure 5C). These results indicate that the expression of TAZ increased in HSCs that had lost their adherens junctions with hepatocytes.

Recent studies show that the Hippo pathway is involved in HSC activation. ${ }^{33,34}$ Therefore, hypothesized that the adherens junctions between hepatocytes and HSCs maintain HSC quiescence through contact inhibition that suppresses YAP/TAZ signaling. To verify this hypothesis, we mimicked E-cadherin-mediated contact inhibition in culture. Mouse primary HSCs, which express E-cadherin for several days after isolation, were cultured on E-cadherincoated dishes to create E-cadherin-mediated adherens junctions. Observation by phase-contrast microscope revealed that mouse HSCs on E-cadherin-coated dishes maintained a quiescent morphology, including multiple thin elongate processes that extended radially from the cell body, ${ }^{35}$ whereas HSCs on uncoated dishes displayed an activation phenotype that included cell spreading and loss of dendritic process ${ }^{36}$ (Figure 5D). In addition, the expression of TAZ and activation markers of HSCs was decreased in mouse HSCs cultured on E-cadherin-coated dishes compared with HSCs cultured on uncoated dishes (Figure 5E). As with mouse primary HSCs, culture of E-cadherin overexpressing human HHSteCs on E-cadherin-coated dishes suppressed the expression of TAZ in a dose-dependent manner, which was paralleled by downregulation of activation markers of HSCs (Figure 5F). Even in E-cadherin-overexpressing HHSteCs, which were cultured as spheroids to reconstruct E-cadherin-mediated adherens junctions, suppression of HSC activation markers down-regulated TAZ expression (Figure 5G).

We next examined the influence of inhibiting E-cadherin-mediated adherens junctions using an E-cadherin neutralizing antibody. The addition of an E-cadherin neutralizing antibody to spheroids of cultured mouse HSCs induced activation markers of HSCs and the enhancement of downstream gene expression of the YAP/TAZ pathway (Figure $5 \mathrm{H}$ ). This outcome suggests that the homophilic binding of E-cadherin regulates HSC activation by downregulating the Hippo pathway.

\section{TAZ Directly and Indirectly Regulates HSCs Activation}

Previous studies have implicated TAZ in HSC activation ${ }^{34,37}$; however, the direct regulation of TAZ in HSCs has not been fully elucidated. Therefore, we overexpressed TAZ in HHSteCs to investigate whether TAZ directly regulated HSC activation TAZ overexpression led to the up-regulation of the

\footnotetext{
Figure 4 The initiation of hepatic stellate cells (HSCs) occurred independently from the stimulation of exogenous initiation factors. A: Liver sections at the indicated time points after carbon tetrachloride $\left(\mathrm{CCl}_{4}\right)$ injection were stained with hematoxylin and eosin. Infiltration of inflammatory cells was present at 12 hours and 24 hours (arrows) but not at 6 hours after $\mathrm{CCl}_{4}$ administration. Inset figures show the enlarged image. B: Immunohistochemical staining for Ly6G, a specific marker of neutrophils, is shown in the left panel. For quantitative analysis, three images captured at every indicated time point, and the number of positive cells for Ly6G per field of each view, were counted (right panel). C: mRNA expression of exogenous stimulation factors for HSC activation and Acta2 in liver. $\mathrm{CCl}_{4}\left(5 \mu \mathrm{L} / \mathrm{g}\right.$ body weight) was injected into mice, and mRNA was isolated from the whole liver at the indicated time points after $\mathrm{CCl}_{4}$ injection. Gene expression for acute inflammatory cytokines that contribute to HSC initiation, including Tnfa, Il1b, Il6, Tgfb, and Pdgf, and an activation marker of HSCs, Acta2, were analyzed by real-time quantitative PCR (qPCR). D: Human HSC line (HHSteC) cells transfected with epithelial cadherin (E-cadherin) or control cells were cultured as spheroids for 48 hours. The top left panel shows control cells and the top right panel shows E-cadherin-transfected HHSteC cells. Then, the expression of transforming growth factor beta (TGF- $\beta$ ) receptor 2 was examined by qPCR (bottom panel). E-G: The influence of E-cadherin-mediated adherens junctions on TGF- $\beta$-Smad2/3 pathway. E: HHSteC cells transfected with E-cadherin or control cells were cultured as spheroid for 48 hours. The HHSteC cells were then stimulated with $10 \mathrm{ng} / \mathrm{mL}$ TGF- $\beta$ for each indicated time. Proteins were isolated at the indicated time points, and the phosphorylation of Smad2 and Smad3 was evaluated by using Western blot analysis. F: HHSteC cells transfected with E-cadherin or control cells were cultured as spheroids for 24 hours. The HHSteC cells were then cultured with $10 \mathrm{ng} / \mathrm{mL}$ TGF- $\beta$ for 48 hours. The gene expression of SNAI1 and CDH2 were analyzed according to qPCR. G: $\alpha$-Smooth muscle actin $(\alpha-S M A)$ expression was investigated by using Western blot analysis. A representative image of three independent experiments is shown in the left panel. For quantification, the band density of $\alpha$-SMA was analyzed and normalized by glyceraldehyde-3-phosphate dehydrogenase (GAPDH) density (right panel). Data are expressed as means \pm SD (B-G). $n=3(\mathbf{A}$ and $\mathbf{C}) ; n=6(\mathbf{D}) ; n=4(\mathbf{F})$. ${ }^{*} P<0.05$ versus 0 ; ${ }^{\dagger} P<0.01$. Scale bars: $100 \mu \mathrm{m}$ (A and B); $50 \mu \mathrm{m}$ (D). 0/E, overexpressed.
} 

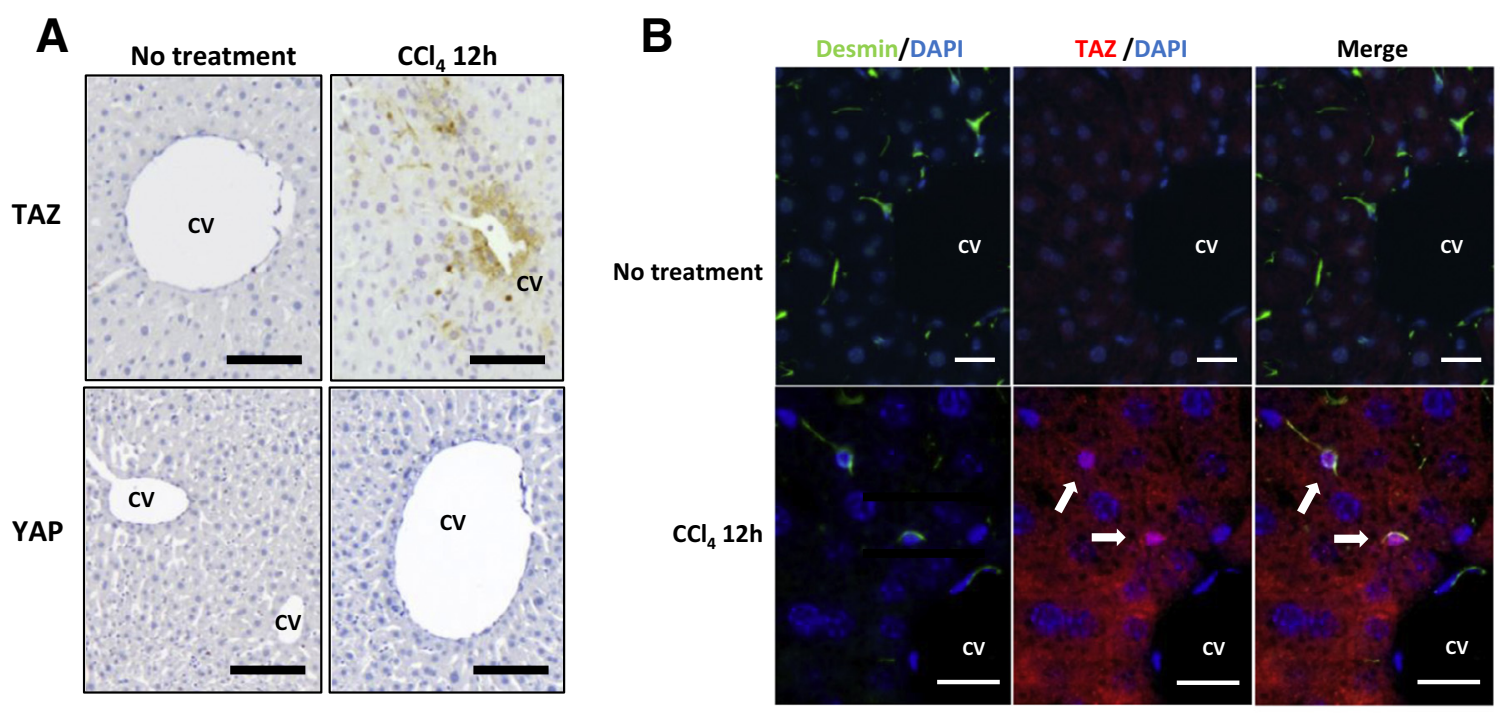

C
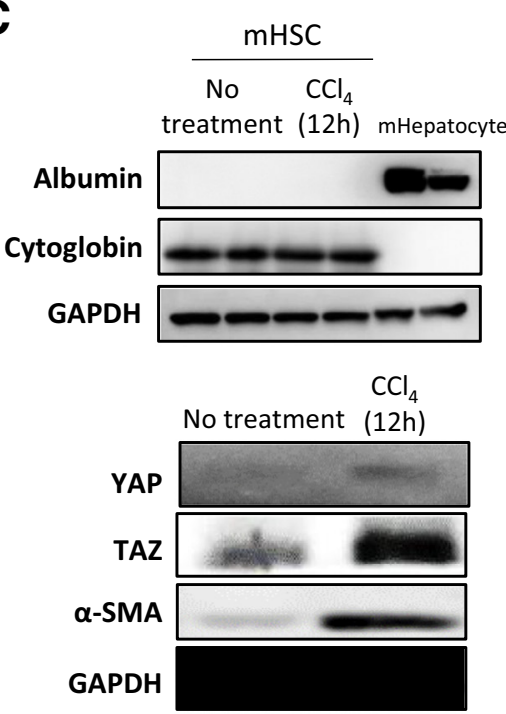

D

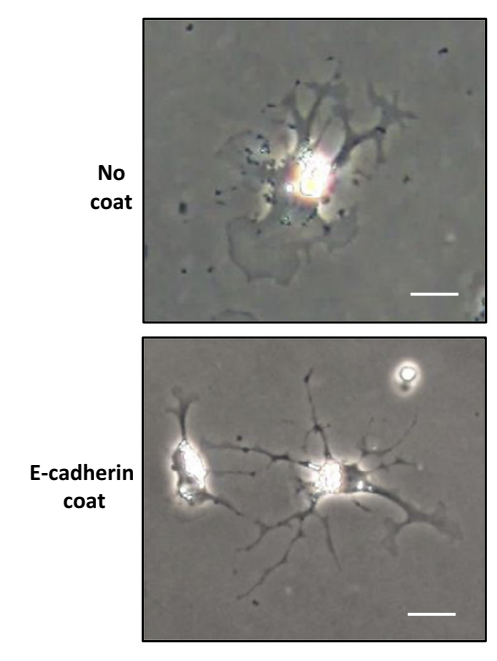

G

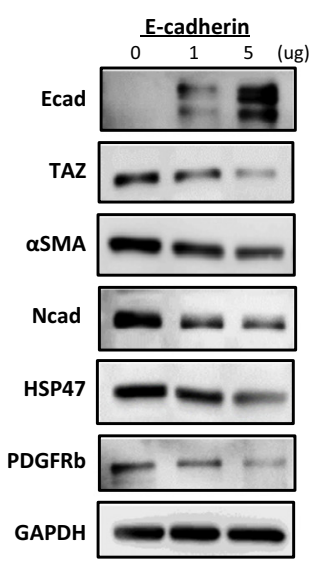

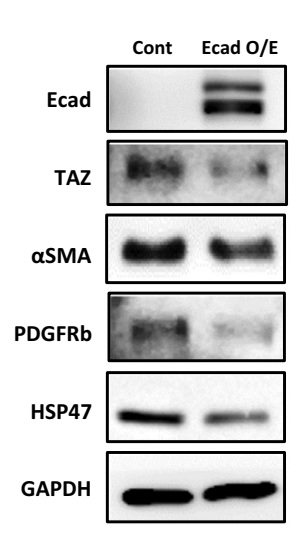

E

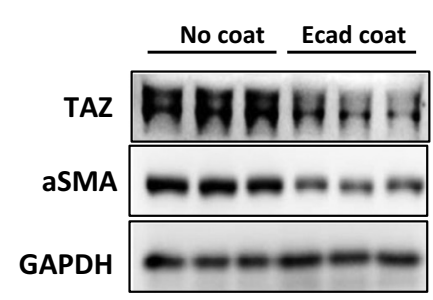

H
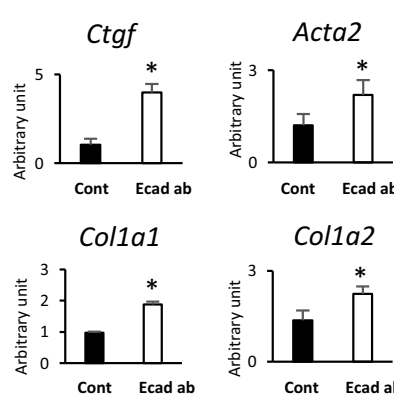

Colla2
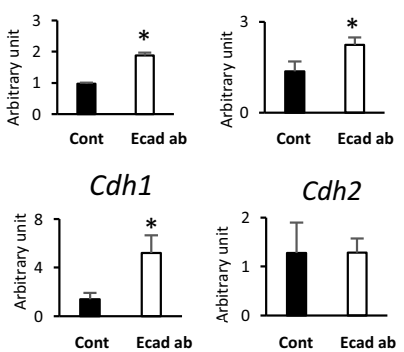
perpetuation markers of HSCs, suggesting that the enhancement of TAZ expression due to the loss of adherens junctions is an initiation factor in HSC activation (Figure 6A). Interestingly, in addition to YAP/TAZ pathway-modulated genes such as $A C T A 2$ and $C T G F$, the downstream genes of TGF- $\beta$ signaling, including PDGFRB and COL1A1, ${ }^{38,39}$ were induced by TAZ overexpression, which was also associated with the increase in TGFB1 gene expression (Figure 6B). Conversely, the expression of TGFB1 in HHSteCs was significantly decreased by reconstitution of E-cadherin-mediated adherens junctions (Figure $6 \mathrm{C}$ ). TGF- $\beta$, which is mainly produced by activated HSCs in mouse fibrosis models, ${ }^{40}$ acts as an autocrine-positive regulator for ECM production $^{40,41}$ and up-regulates $T G F B 1$ gene expression in HHSteCs; the expression level of its receptor TGFBR2 was not changed (Figure 6D). Moreover, after the enhancement of YAP/TAZ expression, TGF- $\beta$ expression in isolated mouse HSCs was increased along with their spontaneous activation (Figure 6E). A previous study reported a decrease in TGF- $\beta$ expression after YAP and TAZ siRNA treatment in NIH3T3 cells. ${ }^{42}$ We therefore hypothesized that TGF- $\beta$ expression increased as a result of HSC activation by YAP/TAZ enhancement, followed by the up-regulation of TGF$\beta$-modulated genes in an autocrine manner. To verify this hypothesis, we examined the expression level of TGF- $\beta$ in the culture supernatants of E-cadherin or TAZ-overexpressed HHSteCs. The reconstruction of E-cadherin-mediated adherens junctions decreased TGF- $\beta$ secretion. Conversely, overexpression of TAZ stimulated TGF- $\beta$ production in the HHSteCs (Figure 6F), indicating that besides direct regulation, TAZ indirectly activates HSCs via enhancement of TGF- $\beta$ production in an autocrine manner.

These results clarify the relationship among E-cadherin-mediated adherens junctions between HSCs and hepatocytes, the role of TAZ, and the activation of HSCs. In normal liver, homophilic E-cadherin binding suppresses TAZ expression, which contributes to maintaining HSC quiescence. In contrast, the loss of this binding in injured liver leads to an increase of TAZ expression and HSC activation (Figure 6G).

\section{Discussion}

This study identifies E-cadherin at the spines of quiescent HSCs as a key component of the adherens junctions between hepatocytes and HSCs. This interaction critically regulates HSC activation through discrete intracellular events that engage YAP/TAZ. Concurrently, isoform switching from Ecadherin to neural cadherin leads to the formation of a complex with platelet-derived growth factor-receptor. ${ }^{43}$ The activation of HSCs by the loss of adherens junctions may lead to loss of contact inhibition, thereby promoting migration and responsiveness to extracellular signals. Based on morphologic observations, Gressner and Lahme's ${ }^{44}$ prediction from over two decades ago that spines in HSCs represent sites of contact inhibition is reinforced by our current findings. Although our studies focused on changes in the Hippo pathway and its effectors (YAP/TAZ), there are also other signals downstream of E-cadherin-mediated contact inhibition, including Wnt and growth signaling pathways. ${ }^{45}$ In addition to rodents, the loss of the adherens junctions has been observed in human liver. Using electron microscopy, Mak and Lieber ${ }^{46}$ investigated human alcoholic steatosis and cirrhosis. In alcoholic steatosis, HSCs with abundant retinoid droplets comprised the adherens junctions linking the cells to surrounding hepatocytes. In the cirrhotic liver, however, HSC lipid content decreased, and their adherens junctions with hepatocytes disappeared, along with the accumulation of collagen in the space of Disse. Although a cause-effect relationship between the loss of adherens junctions with hepatocytes and initiation of HSCs activation in human liver is not fully established, it is clear that HSC loss of adherens junctions with hepatocytes is a feature of the fibrotic liver.

Our findings have also uncovered a mechanism underlying spontaneous activation of rodent HSCs when placed in culture

\footnotetext{
Figure 5 Homophilic binding of epithelial cadherin (E-cadherin) attenuates hepatic stellate cell (HSC) activation by down-regulation of Hippo pathway signaling. A: Liver sections of normal liver or 12 hours after carbon tetrachloride $\left(\mathrm{CCl}_{4}\right)$ injection were immunostained for YAP and TAZ. A representative image around a central vein (CV) is shown. B: Liver sections of untreated or $\mathrm{CCl}_{4}$-injected mice were immunostained for desmin and TAZ for 24 hours followed by DAPI staining for 15 minutes. The expression of TAZ was absent from HSCs expressing the HSC-specific marker desmin in untreated mouse liver (top panels). In contrast, TAZ expression was increased and localized in the nuclei of desmin-positive cells 12 hours after $\mathrm{CCl}_{4}$ injection (white arrows, bottom panels). C: The purity of isolated $\mathrm{HSCS}$ from untreated or $\mathrm{CCl}_{4}$-injected mice was confirmed by Western blot analysis. The expression of cytoglobin but not albumin was observed in the HSCs (top panels). The expression of YAP, TAZ, and $\alpha$-smooth muscle actin ( $\alpha$-SMA) in HSCs isolated from untreated or $\mathrm{CCl}_{4}$-injected mice was examined by Western blot analysis (bottom panels). Two representative images are shown here. D: Isolated mouse HSCs (mHSCs) were cultured on uncoated or E-cadherin-coated dishes for 48 hours. The morphology of HSCs was examined by using a phase-contrast microscope. E: Isolated mouse HSCs were cultured on uncoated or E-cadherin-coated dishes for 48 hours. The expression of TAZ and the HSC activation marker $\alpha$-SMA were examined by using Western blot analysis. F: Human HSC line cells transfected with E-cadherin or control cells were cultured on E-cadherin-coated dishes for 48 hours. The expression of HSC activationrelated molecules was investigated by using Western blot analysis. G: Human HSC line cells transfected with E-cadherin or control cells were cultured as spheroid for 48 hours. The expression of HSC activation-related molecules was investigated by using Western blot analysis. H: The inhibitory effect of Ecadherin-mediated adherens junctions was examined by using an E-cadherin neutralizing antibody. Isolated mHSCs were cultured as spheroid to reconstruct E-cadherin-mediated binding in the presence or absence of E-cadherin neutralization antibody $(10 \mu \mathrm{g} / \mathrm{mL})$. The gene expression of activation markers of HSCs, including downstream of YAP/TAZ pathway such as Ctgf and Acta2, were then examined by real-time quantitative PCR. Data are expressed as means \pm SD (H). $n=4$ (C); $n=3$ (D and H). ${ }^{*} P<0.05$ versus cont. Scale bars: $100 \mu \mathrm{m}(\mathbf{A}) ; 25 \mu \mathrm{m}$ (B); $50 \mu \mathrm{m}$ (D). Ecad ab, E-cadherin neutralization antibody; GAPDH, glyceraldehyde-3-phosphate dehydrogenase; HSP47, heat shock protein 47; mHepatocyte; mouse hepatocyte; Ncad, neural cadherin; 0/E, overexpressed; Pcad, placental cadherin.
} 
A

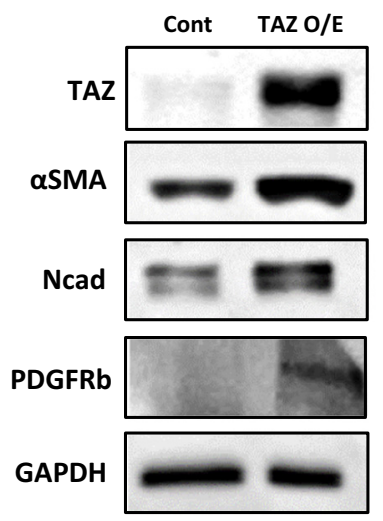

C
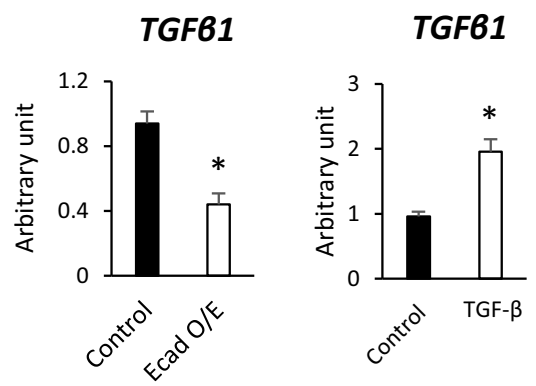

$\mathbf{F}$

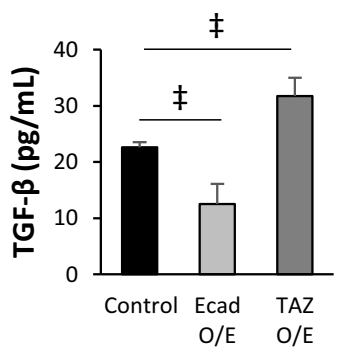

$\mathbf{G}$
B

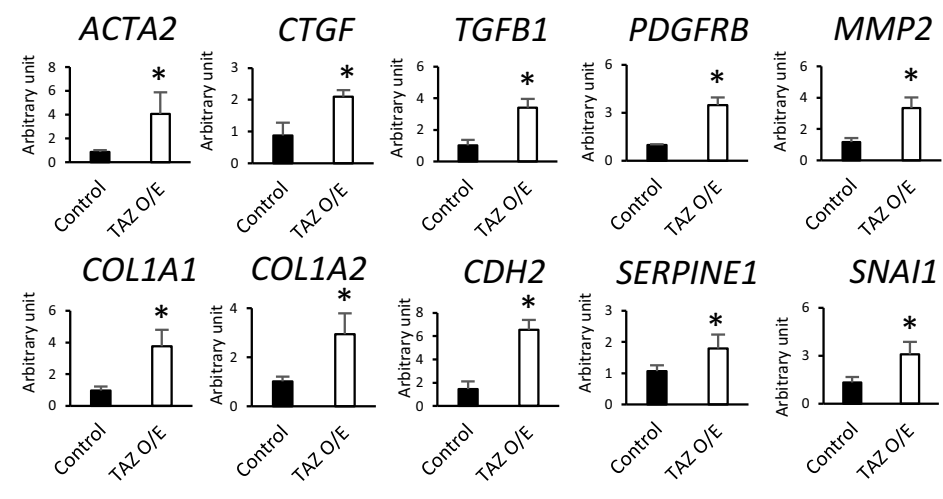

E

TGFBR2
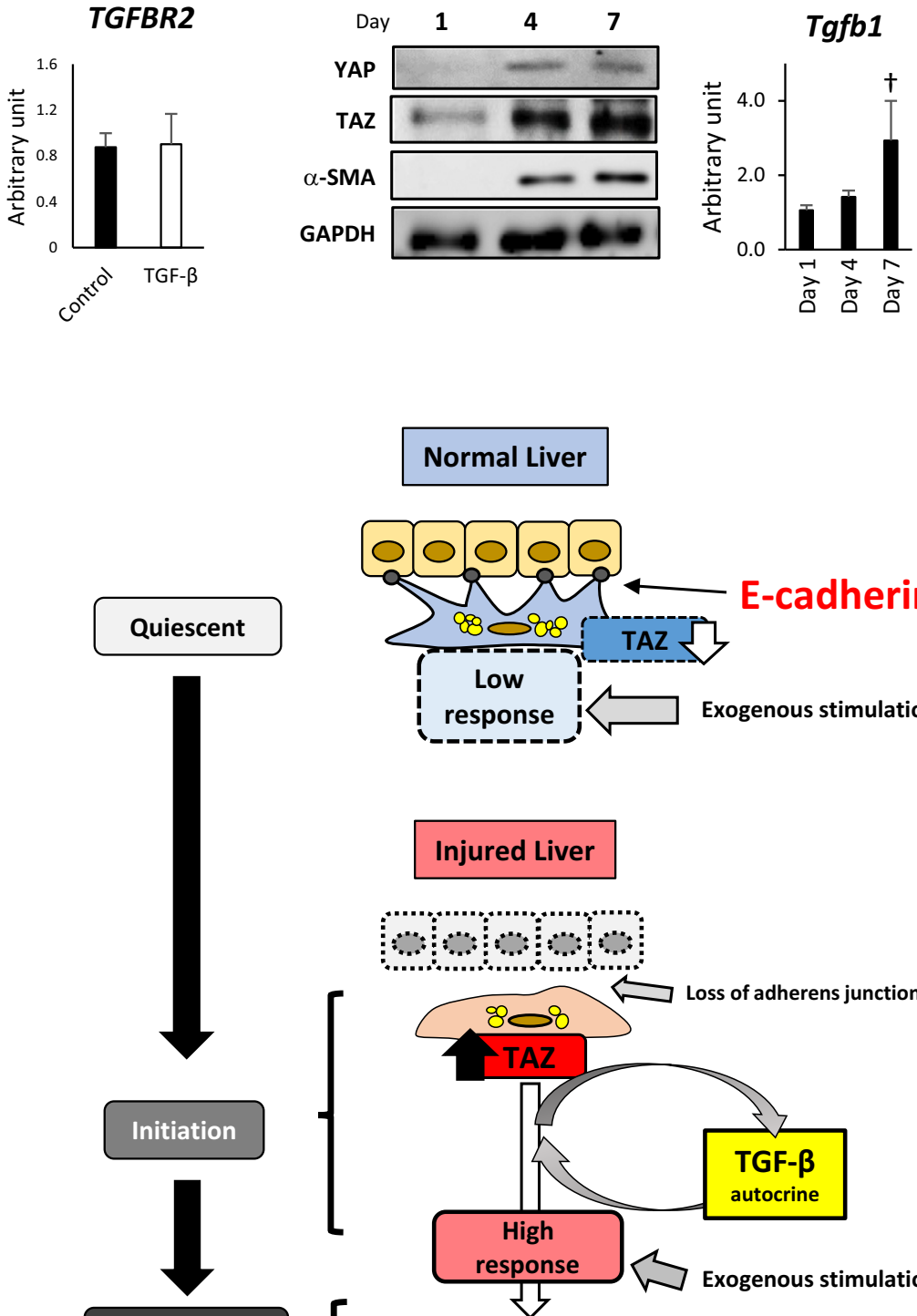

Perpetuation
Normal Liver

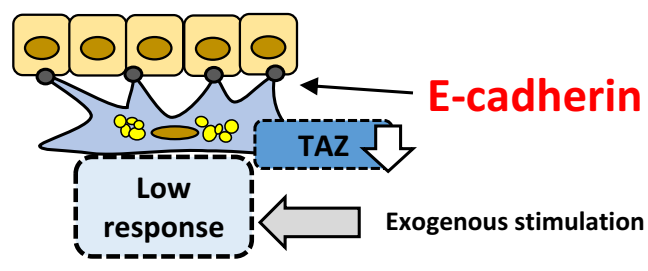

Injured Liver

:
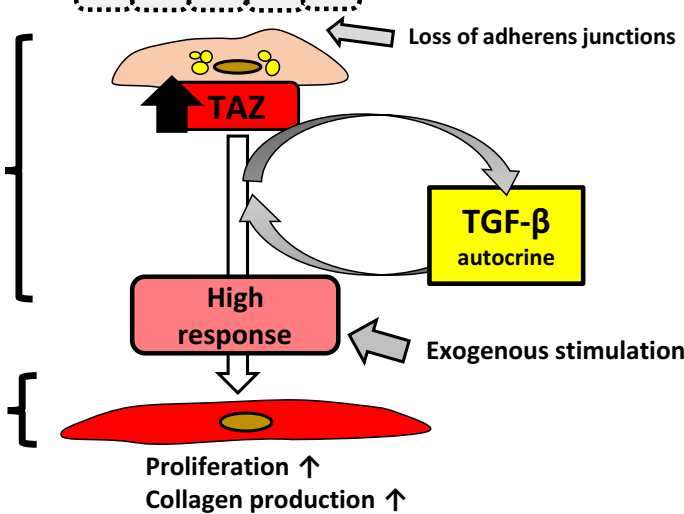
on plastic. The initiation of HSC activation by the upregulation of TAZ induced TGF- $\beta$ expression, consistent with previous findings that secretion of TGF- $\beta$ by isolated HSCs in culture gradually increases in a time-dependent manner. ${ }^{47}$ In addition to HSCs, other cells, including macrophages and LSECs, secrete TGF- $\beta$ during liver injury. Our current results indicate that TGF- $\beta$ activity diminished in HSCs that steadily establish their adherens junctions with hepatocytes, even in the presence of TGF- $\beta$ secreted by these cells. A previous study showed that E-cadherin inhibits Smad2/3 phosphorylation. ${ }^{24}$ Furthermore, the inhibition of YAP/TAZ signaling reduces the phosphorylation of Smad2/ $3,{ }^{37,48,49}$ suggesting that E-cadherin-mediated adhesion of HSCs to hepatocytes not only maintains their quiescence but also attenuates TGF- $\beta$ signaling.

We suggest that a group of LSECs and hepatocytes in contact with one HSC could be considered as a stellate cell unit or "stellon" and propose that the stellon constitutes a functional unit distinct from the hepatic lobule. Viewing our data in the context of a stellon provides a different concept that aligns with the cellular architecture of the liver. Specifically, one HSC surrounds several LSECs and adheres to 20 to 40 hepatocytes via its spines. ${ }^{14}$ Even if one or two hepatocytes are deleted from a hepatic cord, the initiation of HSC activation might not occur because other adherens junctions remain intact. However, in persistent liver injury, the loss of most adherens junctions between HSCs and hepatocytes through broad injury of hepatocytes promotes initiation of HSC activation. HSCs lose their adherens junctions to detach from hepatocytes, which allows them to acquire YAP/TAZ-dependent migration, proliferation, and the production of ECM.

Human HSCs lines, HHSteCs and LX2, are often used for in vitro analysis of HSC biology. However, E-cadherin was barely expressed in these cell lines. Consistent with this finding, the cells were in a state of sustained activation. Thus, these lines would not be suitable to explore mechanisms of initiation of HSC activation. We simulated quiescence in these cell lines by overexpressing E-cadherin or maintainance in spheroid culture. Using this culture model enabled us to seek novel factors driving early HSC activation. In contrast, therapeutically re-expressing E-cadherin could reconstitute adherens junctions to inhibit HSC activation.

We observed that HSCs extend their spines to hepatocytes in the recovery phase of hepatic injury. E-cadherin regulates actin filaments and cell shape,${ }^{50}$ indicating that the HSC spine reflects the cell's dynamics. Interestingly, we have discovered a previously unrecognized structure linking hepatocyte and HSCs. We considered that this fibrous structure possibly contributes to the re-establishment of a linkage between HSCs and hepatocytes. The elucidation of the components and function of the adherens junction by HSCs could reveal important insights into mechanisms of early HSC activation and hepatic fibrosis, and potentially identify novel antifibrotic targets.

The initial trigger for the disappearance of spines in HSCs is still unclear. Many intracellular events occur within hepatocytes immediately after $\mathrm{CCl}_{4}$ administration. Interestingly, HSCs restore their spines during the recovery period from liver injury. We compared the gene expression in the liver of a $\mathrm{CCl}_{4}$-injected rat with that of a normal rat by microarray analysis and identified cell protrusion dynamics-related genes such as cotll ${ }^{51}$ and $f$ In $a,{ }^{52}$ which were confirmed to be localized in activated HSCs (data not shown). Further study is needed to clarify how HSCs recognize this response.

In conclusion, our results suggest that E-cadherin-mediated adherens junctions with hepatocytes maintain HSC quiescence, and detachment from this junction contributes to the activation of HSCs and downstream signaling events that promote hepatic fibrosis.

\section{Author Contributions}

Conceptualization, investigation, visualization, and writing (original draft), H.U.; investigation and formal analysis, H.Y.; conceptualization and formal analysis, T.M.; investigation, N.K. and K. Inoue; investigation and writing (original draft), Y.H.; conceptualization, investigation, and writing (original draft), K.W.; resources and investigation, T.S.; conceptualization and writing (review and editing), S.L.F.; and conceptualization, supervision, writing (original draft), and project administration, K. Ikeda.

\footnotetext{
Figure 6 The relationship among epithelial cadherin (E-cadherin)-mediated adherens junctions between hepatocytes and hepatic stellate cells (HSCs), TAZ expression, and the activation of HSCs. A and B: The direct effect of TAZ overexpression in HSCs on its activation was examined. Human HSC line (HHSteC) cells overexpressed with TAZ or control cells were cultured as spheroid for 48 hours. The influence of TAZ overexpression on HSC activation was investigated by Western blot analysis (A) and real-time quantitative PCR (qPCR) (B). C-F: The relationship among E-cadherin-mediated adherens junction, YAP/TAZ pathway, and autocrine activation of HSCs by transforming growth factor beta (TGF- $\beta$ ). C: HHSteC cells overexpressing E-cadherin or control cells were cultured as spheroids for 48 hours. Gene expression of TGFB1 was analyzed by qPCR. D: HHSteC cells were stimulated by TGF- $\beta$ ( $10 \mathrm{ng} / \mathrm{mL}$ ) for $48 \mathrm{hours}$. Gene expression of TGFB1 and TGFBR2 was then analyzed by qPCR. E: Mouse HSCs were cultured on plastic dishes, and protein or mRNA was isolated at the indicated time points and then evaluated by using Western blot analysis (left panel) and qPCR (right panel). F: E-cadherin or TAZ was transfected in HHSteC cells. The cells were cultured as spheroids for 48 hours. The levels of TGF- $\beta$ in culture medium were then analyzed by ELISA. G: Proposed mechanism of activation of HSCS by dissociation of E-cadherin-containing adherens junctions with hepatocytes. In normal liver, homophilic E-cadherin binding suppresses TAZ expression, which contributes to maintaining HSC quiescence. In contrast, the loss of this binding in injured liver leads to an increase of TAZ expression and autocrine stimulation by TGF- $\beta$, resulting in HSC activation. Data are expressed as means \pm SD (B-F). $n=3(\mathbf{A}$ and $\mathbf{B}) ; n=6(\mathbf{C}$ and $\mathbf{F}) ; n=4(\mathbf{D}$ and $\mathbf{E})$; ${ }^{*} P<0.05$ versus control; ${ }^{\dagger} P<0.05$ versus day $1 ;{ }^{\ddagger} P<0.05 . \alpha$-SMA, $\alpha$-smooth muscle actin; GAPDH, glyceraldehyde-3-phosphate dehydrogenase; $0 / \mathrm{E}$, overexpressed.
} 


\section{References}

1. Troeger JS, Mederacke I, Gwak GY, Dapito DH, Mu X, Hsu CC, Pradere JP, Friedman RA, Schwabe RF: Deactivation of hepatic stellate cells during liver fibrosis resolution in mice. Gastroenterology 2012, 143:1073-1083.e22

2. Iredale JP, Benyon RC, Pickering J, McCullen M, Northrop M, Pawley S, Hovell C, Arthur MJ: Mechanisms of spontaneous resolution of rat liver fibrosis. Hepatic stellate cell apoptosis and reduced hepatic expression of metalloproteinase inhibitors. J Clin Invest 1998 , 102:538-549

3. Fattovich G, Stroffolini T, Zagni I, Donato F: Hepatocellular carcinoma in cirrhosis: incidence and risk factors. Gastroenterology 2004, 127 Suppl 1:S35-S50

4. Friedman SL: Hepatic stellate cells: protean, multifunctional, and enigmatic cells of the liver. Physiol Rev 2008, 88:125-172

5. Tsuchida T, Friedman SL: Mechanisms of hepatic stellate cell activation. Nat Rev Gastroenterol Hepatol 2017, 14:397-411

6. Borkham-Kamphorst E, van Roeyen CRC, Ostendorf T, Floege J, Gressner AM, Weiskirchen R: Pro-fibrogenic potential of PDGF-D in liver fibrosis. J Hepatol 2007, 46:1064-1074

7. Pinzani M: PDGF and signal transduction in hepatic stellate cells. Front Biosci 2002, 7:d1720-d1726

8. Breitkopf K, Godoy P, Ciuclan L, Singer MV, Dooley S: TGFbeta/Smad signaling in the injured liver. Z Gastroenterol 2006, 44: $57-66$

9. Gressner AM, Weiskirchen R, Breitkopf K, Dooley S: Roles of TGFbeta in hepatic fibrosis. Front Biosci 2002, 7:d793-d807

10. Iredale JP, Murphy G, Hembry RM, Friedman SL, Arthur MJ: Human hepatic lipocytes synthesize tissue inhibitor of metalloproteinases-1. Implications for regulation of matrix degradation in liver. J Clin Invest 1992, 90:282-287

11. Friedman SL, Roll FJ, Boyles J, Arenson DM, Bissell DM: Maintenance of differentiated phenotype of cultured rat hepatic lipocytes by basement membrane matrix. J Biol Chem 1989, 264: 10756-10762

12. Lim YS, Lee HC, Lee HS: Switch of cadherin expression from E- to N-type during the activation of rat hepatic stellate cells. Histochem Cell Biol 2007, 127:149-160

13. Wake K: Cell-cell organization and functions of 'sinusoids' in liver microcirculation system. J Electron Microsc (Tokyo) 1999, 48: 89-98

14. Wake K: Hepatic stellate cells: three-dimensional structure, localization, heterogeneity and development. Proc Jpn Acad Ser B Phys Biol Sci 2006, 82:155-164

15. Kristensen DB, Kawada N, Imamura K, Miyamoto Y, Tateno C, Seki S, Kuroki T, Yoshizato K: Proteome analysis of rat hepatic stellate cells. Hepatology 2000, 32:268-277

16. Mabuchi A, Mullaney I, Sheard PW, Hessian PA, Mallard BL, Tawadrous MN, Zimmermann A, Senoo H, Wheatley AM: Role of hepatic stellate cell/hepatocyte interaction and activation of hepatic stellate cells in the early phase of liver regeneration in the rat. J Hepatol 2004, 40:910-916

17. Yagi T, Takeichi M: Cadherin superfamily genes: functions, genomic organization, and neurologic diversity. Genes Dev 2000, 14: $1169-1180$

18. Nollet F, Kools P, van Roy F: Phylogenetic analysis of the cadherin superfamily allows identification of six major subfamilies besides several solitary members. J Mol Biol 2000, 299:551-572

19. Takeichi M: Morphogenetic roles of classic cadherins. Curr Opin Cell Biol 1995, 7:619-627

20. Kawada N: Cytoglobin as a marker of hepatic stellate cell-derived myofibroblasts. Front Physiol 2015, 6:329

21. Kawada N, Kuroki T, Kobayashi K, Inoue M, Nakatani K, Kaneda K, Nagata K: Expression of heat-shock protein 47 in mouse liver. Cell Tissue Res 1996, 284:341-346
22. Han YP, Zhou L, Wang J, Xiong S, Garner WL, French SW, Tsukamoto H: Essential role of matrix metalloproteinases in interleukin-1-induced myofibroblastic activation of hepatic stellate cell in collagen. J Biol Chem 2004, 279:4820-4828

23. Yokoi Y, Namihisa T, Kuroda H, Komatsu I, Miyazaki A, Watanabe S, Usui K: Immunocytochemical detection of desmin in fat-storing cells (Ito cells). Hepatology 1984, 4:709-714

24. Cho IJ, Kim YW, Han CY, Kim EH, Anderson RA, Lee YS, Lee CH, Hwang SJ, Kim SG: E-cadherin antagonizes transforming growth factor [beta]1 gene induction in hepatic stellate cells by inhibiting RhoA-dependent Smad3 phosphorylation. Hepatology 2010, 52: 2053-2064

25. Koyama Y, Brenner DA: Liver inflammation and fibrosis. J Clin Invest 2017, 127:55-64

26. Knittel T, Müller L, Saile B, Ramadori G: Effect of tumour necrosis factor-alpha on proliferation, activation and protein synthesis of rat hepatic stellate cells. J Hepatol 1997, 27:1067-1080

27. Reiter FP, Wimmer R, Wottke L, Artmann R, Nagel JM, Carranza MO, Mayr D, Rust C, Fickert P, Trauner M, Gerbes AL, Hohenester S, Denk GU: Role of interleukin-1 and its antagonism of hepatic stellate cell proliferation and liver fibrosis in the Abcb4(-/-) mouse model. World J Hepatol 2016, 8:401-410

28. Yaping Z, Ying W, Luqin D, Ning T, Xuemei A, Xixian Y: Mechanism of interleukin-1[beta]-induced proliferation in rat hepatic stellate cells from different levels of signal transduction. APMIS 2014, 122:392-398

29. Xiang DM, Sun W, Ning BF, Zhou TF, Li XF, Zhong W, Cheng Z, Xia MY, Wang X, Deng X, Wang W, Li HY, Cui XL, Li SC, Wu B, Xie WF, Wang HY, Ding J: The HLF/IL-6/STAT3 feedforward circuit drives hepatic stellate cell activation to promote liver fibrosis. Gut 2018, 67:1704-1715

30. Fagotto F, Gumbiner BM: Cell contact-dependent signaling. Dev Biol 1996, 180:445-454

31. Perrais M, Chen X, Perez-Moreno M, Gumbiner BM: E-cadherin homophilic ligation inhibits cell growth and epidermal growth factor receptor signaling independently of other cell interactions. Mol Biol Cell 2007, 18:2013-2025

32. Kim NG, Koh E, Chen X, Gumbiner BM: E-cadherin mediates contact inhibition of proliferation through Hippo signaling-pathway components. Proc Natl Acad Sci U S A 2011, 108:11930-11935

33. Mannaerts I, Leite SB, Verhulst S, Claerhout S, Eysackers N, Thoen LFR, Hoorens A, Reynaert H, Halder G, van Grunsven LA: The Hippo pathway effector YAP controls mouse hepatic stellate cell activation. J Hepatol 2015, 63:679-688

34. Konishi T, Schuster RM, Lentsch AB: Proliferation of hepatic stellate cells, mediated by YAP and TAZ, contributes to liver repair and regeneration after liver ischemia-reperfusion injury. Am J Physiol Gastrointest Liver Physiol 2018, 314:G471-G482

35. Yee HF Jr: Rho directs activation-associated changes in rat hepatic stellate cell morphology via regulation of the actin cytoskeleton. Hepatology 1998, 28:843-850

36. Kawada N, Harada K, Ikeda K, Kaneda K: Morphological study of endothelin-1-induced contraction of cultured hepatic stellate cells on hydrated collagen gels. Cell Tissue Res 1996, 286:477-486

37. Wang Y, Tu K, Liu D, Guo L, Chen Y, Li Q, Maiers JL, Liu Z, Shah VH, Dou C, Tschumperlin D, Voneschen L, Yang R, Kang N: p300 Acetyltransferase is a cytoplasm-to-nucleus shuttle for SMAD2/3 and TAZ nuclear transport in transforming growth factor [beta]-stimulated hepatic stellate cells. Hepatology 2019, 70: $1409-1423$

38. Boucher P, Li WP, Matz RL, Takayama Y, Auwerx J, Anderson RGW, Herz J: LRP1 functions as an atheroprotective integrator of TGFbeta and PDFG signals in the vascular wall: implications for Marfan syndrome. PloS One 2007, 2:e448

39. García-Trevijano ER, Iraburu MJ, Fontana L, Domínguez-Rosales JA, Auster A, Covarrubias-Pinedo A, Rojkind M: Transforming growth factor beta1 induces the expression of alpha1(I) procollagen mRNA by 
a hydrogen peroxide-C/EBPbeta-dependent mechanism in rat hepatic stellate cells. Hepatology 1999, 29:960-970

40. Bissell DM, Wang SS, Jarnagin WR, Roll FJ: Cell-specific expression of transforming growth factor-beta in rat liver. Evidence for autocrine regulation of hepatocyte proliferation. J Clin Invest 1995, 96:447-455

41. Gressner AM, Bachem MG: Molecular mechanisms of liver fibrogenesis - a homage to the role of activated fat-storing cells. Digestion 1995, 56:335-346

42. Lee MJ, Byun MR, Furutani-Seiki M, Hong JH, Jung HS: YAP and TAZ regulate skin wound healing. J Invest Dermatol 2014, 134: $518-525$

43. Theisen CS, Wahl JK 3rd, Johnson KR, Wheelock MJ: NHERF links the N-cadherin/catenin complex to the platelet-derived growth factor receptor to modulate the actin cytoskeleton and regulate cell motility. Mol Biol Cell 2007, 18:1220-1232

44. Gressner A, Lahme B: Inhibitory actions of hepatocyte plasma membranes on proliferation, protein- and proteoglycan synthesis of cultured rat fat storing cells. Cells Hepatic Sinusoid 1991, 3:237-241

45. Jeanes A, Gottardi CJ, Yap AS: Cadherins and cancer: how does cadherin dysfunction promote tumor progression? Oncogene 2008, 27:6920-6929

46. Mak KM, Lieber CS: Lipocytes and transitional cells in alcoholic liver disease: a morphometric study. Hepatology 1988, 8:1027-1033
47. Bachem MG, Meyer D, Melchior R, Sell KM, Gressner AM: Activation of rat liver perisinusoidal lipocytes by transforming growth factors derived from myofibroblastlike cells. A potential mechanism of selfperpetuation in liver fibrogenesis. J Clin Invest 1992, 89:19-27

48. Szeto SG, Narimatsu M, Lu M, He X, Sidiqi AM, Tolosa MF, Chan L, De Freitas K, Bialik JF, Majumder S, Boo S, Hinz B, Dan Q, Advani A, John R, Wrana JL, Kapus A, Yuen DA: YAP/TAZ are mechanoregulators of TGF-[beta]-Smad signaling and renal fibrogenesis. J Am Soc Nephrol 2016, 27:3117-3128

49. Qin Z, Xia W, Fisher GJ, Voorhees JJ, Quan T: YAP/TAZ regulates TGF-[beta]/Smad3 signaling by induction of Smad7 via AP-1 in human skin dermal fibroblasts. Cell Commun Signal 2018, 16:18

50. Michael M, Yap AS: The regulation and functional impact of actin assembly at cadherin cell-cell adhesions. Semin Cell Dev Biol 2013, 24:298-307

51. Kim J, Shapiro MJ, Bamidele AO, Gurel P, Thapa P, Higgs HN, Hedin KE, Shapiro VS, Billadeau DD: Coactosin-like 1 antagonizes cofilin to promote lamellipodial protrusion at the immune synapse. PLoS One 2014, 9:e85090

52. Maceyka M, Alvarez SE, Milstien S, Spiegel S: Filamin A links sphingosine kinase 1 and sphingosine-1-phosphate receptor 1 at lamellipodia to orchestrate cell migration. Mol Cell Biol 2008, 28: $5687-5697$ 